\title{
Downsizing metal-organic frameworks by bottom-up and top-down methods
}

\author{
Ken Aldren S. Usman ${ }^{1,2,3}$, James W. Maina ${ }^{2}$, Shayan Seyedin $\mathbb{E}^{2}$, Marlon T. Conato ${ }^{3}$, Leon M. PayawanJr. ${ }^{3}$ \\ Ludovic F. Dumée $\mathbb{D}^{2}$ and Joselito M. Razal ${ }^{2}$
}

\begin{abstract}
Downsizing metal-organic framework (MOF) crystals into the nanoregime offers a promising approach to further benefit from their inherent versatile pore structures and surface reactivity. In this article, downsizing is referred to as the deliberate production of typical large MOF crystals into their nanosized versions. Here, we discuss various strategies towards the formation of crystals below $100 \mathrm{~nm}$ and their impact on the nano-MOF crystal properties. Strategies include an adjustment of the synthesis parameters (e.g., time, temperature, and heating rate), surface modification, ligand modulation, control of solvation during crystal growth and physical grinding methods. These approaches, which are categorized into bottom-up and top-down methods, are also critically discussed and linked to the kinetics of MOF formation as well as to the homogeneity of their size distribution and crystallinity. This collection of downsizing routes allows one to tailor features of MOFs, such as the morphology, size distribution, and pore accessibility, for a particular application. This review provides an outlook on the enhanced performance of downsized MOFs along with their potential use for both existing and novel applications in a variety of disciplines, such as medical, energy, and agricultural research.
\end{abstract}

\section{Introduction}

Metal-organic frameworks (MOFs) are crystals consisting of metal ions/coordination complexes connected by organic ligands into periodic structures ${ }^{1,2}$. Due to their structural motifs, cage-like structures, and high internal surface area and porosity, MOF materials and their composites have shown very promising potential across a wide array of applications, such as sensing ${ }^{3}$, gas storage and separation ${ }^{4}$, light harvesting ${ }^{5}$, molecular sieving ${ }^{6}$, controlled fertilizer release ${ }^{7,8}$, and drug delivery ${ }^{9-11}$. MOFs have also been considered promising catalysts/ hosts for electrochemical energy conversion ${ }^{12}$ since compared to the structure of nanoporous carbon benchmarks, MOFs offer more uniform pore structures with

\footnotetext{
Correspondence: Ludovic F. Dumée (ludovic.dumee@deakin.edu.au)

${ }^{1}$ Natural Sciences Research Institute, College of Science, University of the Philippines, Diliman, Quezon City 1101, Philippines

${ }^{2}$ Deakin University, Institute for Frontier Materials, Waurn Ponds, Geelong, VIC 3216, Australia

Full list of author information is available at the end of the article
}

higher specific surface areas and electrochemically active metal ions/complexes across their backbone ${ }^{12}$. These properties provide accessible electrochemically active sites and enable efficient mobility of gas molecules and ions (e.g., nitrates and amines), making them suitable for sensing applications ${ }^{12}$.

When reduced to the nanoregime, MOF crystals can offer significantly enhanced physical and chemical properties compared to their bulk counterparts. Due to their controllable diffusion kinetics and efficient confinement of redox centers, nano-MOFs can be utilized in novel applications where a specific size and shape are required (e.g., sulfur storage for energy conversion applications) ${ }^{13,14}$. The synthesis of MOFs can be tuned by carefully choosing the metal and organic components to generate typically tens to hundreds of micron-sized large and continuous crystals with extremely uniform structures and homogenous properties (e.g., pore metrics, chemical compositions, and adsorption profiles) ${ }^{15}$. Although the pore metrics of the frameworks may be

\section{(c) The Author(s) 2020}

(c) Open Access This article is licensed under a Creative Commons Attribution 4.0 International License, which permits use, sharing, adaptation, distribution and reproduction cc) in any medium or format, as long as you give appropriate credit to the original author(s) and the source, provide a link to the Creative Commons license, and indicate if changes were made. The images or other third party material in this article are included in the article's Creative Commons license, unless indicated otherwise in a credit line to the material. If material is not included in the article's Creative Commons license and your intended use is not permitted by statutory regulation or exceeds the permitted use, you will need to obtain permission directly from the copyright holder. To view a copy of this license, visit http://creativecommons.org/licenses/by/4.0/. 
altered by modulating the length or shape of the organic linkers, growth conditions or the presence of additives during nucleation ${ }^{16}$, challenges associated with limiting MOF crystal growth to the nanoscale regime have hindered their scope of application ${ }^{17}$. Several downsizing techniques that bring MOFs into the nanodomain were shown to offer routes to amplify the natural properties of the MOFs by refining their surface-to-volume ratio and by generating increased volumetric densities of terminal or defective sites ${ }^{17,18}$

Emerging downsized MOF materials have found applications in several areas, such as heterogeneous catalysis ${ }^{17}$, sensor design ${ }^{17}$, biosensing ${ }^{19}$, biomedical imaging ${ }^{19}$, and drug delivery ${ }^{10,19}$. During drug delivery, for instance, the design of novel micro- and/or nanosized carriers for drugs and therapeutic agents has provided solutions to critical drawbacks associated with the solubility, bioavailability, immunocompatibility, and nanotoxicity of carriers and probes $^{20,21}$. Recent studies also revealed that the blood half-life and diffusion kinetics of nanoparticle-based drug delivery systems are size dependent ${ }^{22}$. Transport across biocapillaries and effective cellular uptake require nanometer-sized materials, and the typical bulk crystal size of MOFs is not suited to this type of application. In addition, properties, such as the shape, surface area, and surface charge, play important roles in particle-cell interactions, biodistribution, and pharmacokinetics ${ }^{22}$. Likewise, nano-MOFs were also shown to possess promising properties for sensing applications, where a high surface area and porosity increase the sensitivity and reduce the mass transfer limitations of sensors ${ }^{23}$.

This study reviews current strategies to downsize commonly used micrometer-sized MOFs. Here, we refer to downsizing as the deliberate production of nanosized MOFs from the typical microscale. Although various rational design strategies to produce novel nanosized MOFs were previously reviewed, most of these studies focused on the synthetic design and rational control of MOF nanomorphologies to fabricate novel nanocrystals that ${ }^{10,21-26}$ are geared towards their use in specific applications, such as catalysis ${ }^{22}$, electronics ${ }^{27}$, imaging/ sensing $^{28}$, and biomedical applications ${ }^{29-31}$. At this stage, the current literature still lacks insights into the direct scaling down process of well-known bulk MOFs. The production of nanosized versions of well-established MOFs with well-studied and inherently ideal properties for certain applications (such as number of active sites, pore size, and biocompatibility) could provide improved performance. Therefore, this review article analyzes the direct downsizing strategies of MOFs that have demonstrated desirable properties in their respective applications. The first part includes a brief overview of the general MOF crystallization mechanisms that are critical in understanding the reaction-limited growth routes and the impact of processes and chemicals on the shape and size of MOFs to generate MOF materials at the nanoscale. The discussion on downsizing methods starts with the simplest bottom-up approach of the straightforward adjustment of synthesis parameters. The succeeding section discusses chemical-based crystal growth modification methods, such as microemulsions, ligand variation, and solvents. Top-down approaches, such as physical downsizing methods that are able to effectively reduce the size of MOF crystals, are critically presented last. The impact of each route on the properties and stability of the downsized MOFs is also evaluated, and the properties of the downsized MOFs are compared to those of their bulk counterparts. This review provides researchers with a comprehensive and technical overview of the latest technological solutions to downsize MOFs from the macro- to nanoscale. This review not only discusses the impact of downsizing on MOF properties but also offers, for the first time, a critical discussion on the remaining challenges in the area. In addition, the discussion also includes comments on the cost-effectiveness and scalability of the techniques toward industrial scale applications ranging from catalysis, biomedicine, agriculture, sensing, and energy research, which is beneficial in terms of upscaling since the discussed techniques are mostly straightforward and require the least chemical modifications.

\section{Overview on the nucleation and crystallization process of MOFs}

Understanding the fundamental chemistry of MOF formation is of utmost importance for the systematic control of its crystal structure and morphology during synthesis $^{32}$. Systematically modifying specific stages in the crystallization process, as well as applying physical grinding methods and PSMs, has been successful in the size-controlled synthetic production of zeolites for both small-scale and industrial quantities ${ }^{33,34}$. The use of these modification strategies as synthesis benchmarks could essentially lead to the production of other nanosized crystals, such as MOFs. Similar to that of zeolites, MOF formation also starts with the assembly of inorganic clusters that are eventually bridged together by a tunable organic linker. The early stages of this process (shown in Fig. 1) involve an incubation stage where the metal complex, also known as the secondary building unit (SBU), replaces the original "template" or the structure of anions originally attached to the metal ${ }^{24-26}$. Typically, this process is conducted in hydrothermal/solvothermal conditions, and a period known as the nucleation stage ${ }^{24-26}$ occurs, where interconnected complexes start to form the self-assembled "seeds"/metastable phases. This process is followed by a growth period where the material starts to develop its crystal structure ${ }^{24-26}$. At this step, the solvent molecules trapped within the pores through 


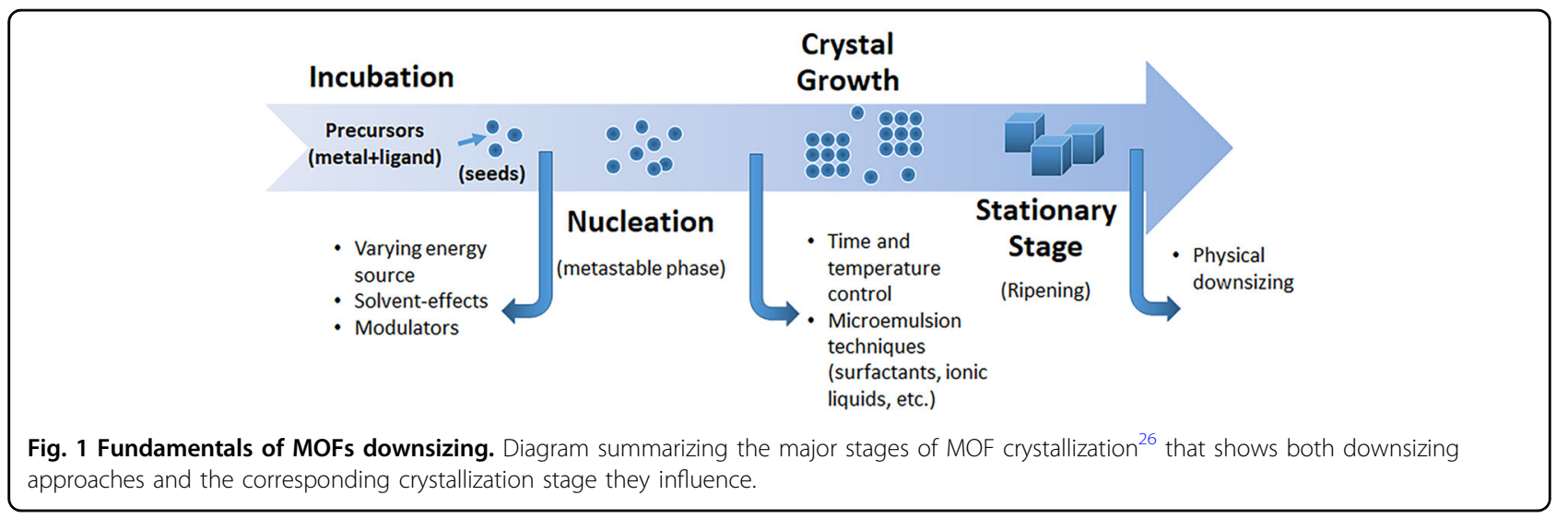

intermolecular interactions with the ligand are critical in balancing the charge of the framework ${ }^{24-26}$. The final stage in MOF formation involves a stationary stage where all crystals in the solution become homogeneous in size and phase ${ }^{26}$. At this stage, all noncrystalline and metastable phases in the solution are consumed. Last, postsynthetic modification (PSM) of MOFs, such as grafting and functionalization of the organic linker, has turned out to be an effective approach for modifying the reactivity of pores, which is beneficial to certain applications, such as catalysis ${ }^{16}$.

The information about the structural evolution of MOFs provides researchers with several windows to introduce modifications that could tune the growth of crystals, as shown in Fig. 1.

\section{Bottom-up methods}

The crystal sizes of MOFs can be fundamentally minimized by controlling their nucleation rate, as it is a widely accepted fact that an increased number of nucleation sites results in small MOF crystal sizes ${ }^{13}$. Here, most of the precursors are consumed prior to the crystal growth stage, which results in a high yield of small crystals. This is achieved by providing energy sources that promote fast nucleation, such as microwave ${ }^{27-29}$ and sonochemical $^{6,30,31}$ methods. A variation in the solvent ${ }^{32,33}$ and addition of modulators ${ }^{34-37}$ have also been reported to enhance nucleation rates for MOFs, resulting in decreased crystal size distributions.

The growth of MOFs can also be minimized by hindering the crystal growth stage to limit the aggregation of seeds. This approach has been successfully reported in several studies where a shorter time $e^{26,38}$ and decreased temperature ${ }^{17,39}$ limit growth of the crystals. Size control has also been demonstrated through microemulsion techniques with the aid of additives, such as surfactants ${ }^{40-43}$ and/or ionic liquids (IL) $)^{44,45}$, which limits the crystal growth of MOFs to the size and shape of the micelle produced by the emulsion.

\section{Additive-free methods via adjustment of synthesis parameters}

This section discusses straightforward additive-free tuning of MOF nucleation and crystal growth. Overall, the most convenient procedures for downsizing MOFs are those with minimum chemical modifications, such as growth control via the adjustment of synthesis parameters. These methods include adjusting the time, temperature, and energy source, such as microwaves and ultrasonication as well as mechanical stress.

\section{Kinetics control-impact of the experimental parameters}

The evolution of MOF particle size is governed by the MOF's crystal growth rate and is a function of time ${ }^{26,38}$. Therefore, a simple and straightforward approach to obtain small MOF crystal sizes is to control the kinetics of crystal formation via a shortened synthesis duration. The crystal growth of MOFs as a function of time was verified through a mechanistic study of zeolitic imidazolate framework-8 (ZIF-8) ${ }^{26}$. This study investigated the different phases of ZIF-8 development (nucleation, crystallization, growth, and stationary periods) and reported an overview of its crystal transformation kinetics. Timedependent evolution of ZIF-8 size was confirmed through TEM analysis of samples synthesized at various durations from 10 min to $24 \mathrm{~h}$. ZIF- 8 crystals evolved from spherical particles with a size of $50 \mathrm{~nm}$ into well-defined polyhedral crystals with sizes of approximately $500 \mathrm{~nm}$, where faceted crystals were formed starting at $60 \mathrm{~min}$ of synthesis. The maximum relative crystallinity was achieved along with increased crystal homogeneity in terms of size and shape within $1 \mathrm{~h}$ of synthesis.

Size control through a decreased crystallization time was also demonstrated through the synthesis of small and monodispersed isoreticular zinc bis(pyrazolate) (Zn-BDP) $\mathrm{MOFs}^{38}$. Particles with an average hydrodynamic diameter of $\sim 105 \mathrm{~nm}$ were formed by limiting the reaction duration to $1 \mathrm{~h}$ compared to $\sim 180 \mathrm{~nm}$ crystals that were produced after $7 \mathrm{~h}$ of synthesis. The particles acquired at 
shorter crystallization times had a lower polydispersity index (PdI) when dispersed in dimethylformamide (DMF) compared to those synthesized at prolonged heating up to $7 \mathrm{~h}$ (PdI of 0.4 vs. 0.6, respectively). Small Zn-BDP MOFs with low PdI values have shown their potential in drug delivery applications, for which dispersed nano-MOFs (synthesized for $1 \mathrm{~h}$ ) also exhibited a stable zeta potential in polar solvents, such as Milli-Q water and phosphatebuffered saline (PBS), even for a prolonged dispersion time (the Milli-Q stable zeta potential was $\sim 22 \mathrm{mV}$ after $30 \mathrm{~s}$ and $-18 \mathrm{mV}$ after $24 \mathrm{~h}$; the PBS stable zeta potential was approximately $-8 \mathrm{mV}$ after $30 \mathrm{~s}$ and $-14 \mathrm{mV}$ after $24 \mathrm{~h})$.

It is also recognized that lowering the reaction temperature combined with decreasing the reaction duration results in a decreased crystal growth rate ${ }^{17,46}$. The effect of varying both the crystallization temperature and time on the crystal growth of MOFs is exhibited in Fig. 2. Varying the synthesis time of Fe-MIL-88A crystals resulted in a gradual evolution of MOF crystals from undefined morphologies with sizes ranging from 100 to $300 \mathrm{~nm}$ to full-grown rods with sizes $>1000 \mathrm{~nm}$ upon extending the reaction to $24 \mathrm{~h}^{39}$. The resulting morphological difference between crystals synthesized for 6 and $24 \mathrm{~h}$ (Fig. 2a) clearly demonstrated the effect of shortening both the crystal growth and stationary stages. This decrease in the synthesis duration resulted in insufficient time for the Fe-MIL-88A crystals to evolve into mature, rod-like crystals. The lowering of the reaction temperature to $65{ }^{\circ} \mathrm{C}$ for crystals synthesized between 0.5 and $24 \mathrm{~h}$ also resulted in particle sizes ranging from 110 to $1050 \mathrm{~nm}$ (Fig. 2b). These values are smaller than those synthesized for the same synthesis durations at $100^{\circ} \mathrm{C}$, which had sizes ranging from 195 to $1460 \mathrm{~nm}$. The effect of combined time and temperature adjustments on MOF crystallization was also evident, with yield values at $100^{\circ} \mathrm{C}$ for $24 \mathrm{~h}$ of up to $63 \pm 3 \%$, which is significantly higher than that of crystals acquired at a lower time and temperature that yielded $<50 \%$ (Fig. 2c).

Similarly, polydispersed Co-based ZIF-67 crystals were produced through careful modification of the synthesis conditions by varying the solvothermal temperature and crystallization time ${ }^{17}$. Bulk ZIF-67 crystals that were $1.7 \mu \mathrm{m}$ in size were prepared using $\mathrm{Co}(\mathrm{OAc})_{2} \cdot 4 \mathrm{H}_{2} \mathrm{O}$ with 2-methylimidazole mixed and heated in ethanol at $120^{\circ} \mathrm{C}$ for 3 days. Crystals that were $800 \mathrm{~nm}$ in size were prepared at $60^{\circ} \mathrm{C}(20 \mathrm{~h})$, while the smallest $300 \mathrm{~nm}$ particles were synthesized at $25^{\circ} \mathrm{C}(20 \mathrm{~h})$. This downsizing strategy resulted in a significantly higher specific surface area for the nanocrystals, with values ranging from 233 to $386 \mathrm{~m}^{2} \mathrm{~g}^{-1}$ compared to $165 \mathrm{~m}^{2} \mathrm{~g}^{-1}$ for the bulk crystals. The rhombic dodecahedral morphology of ZIF-67 crystals was only prominent for the bulk samples, while the nanocrystals (800 and $300 \mathrm{~nm}$ in size) were spherical. The

a
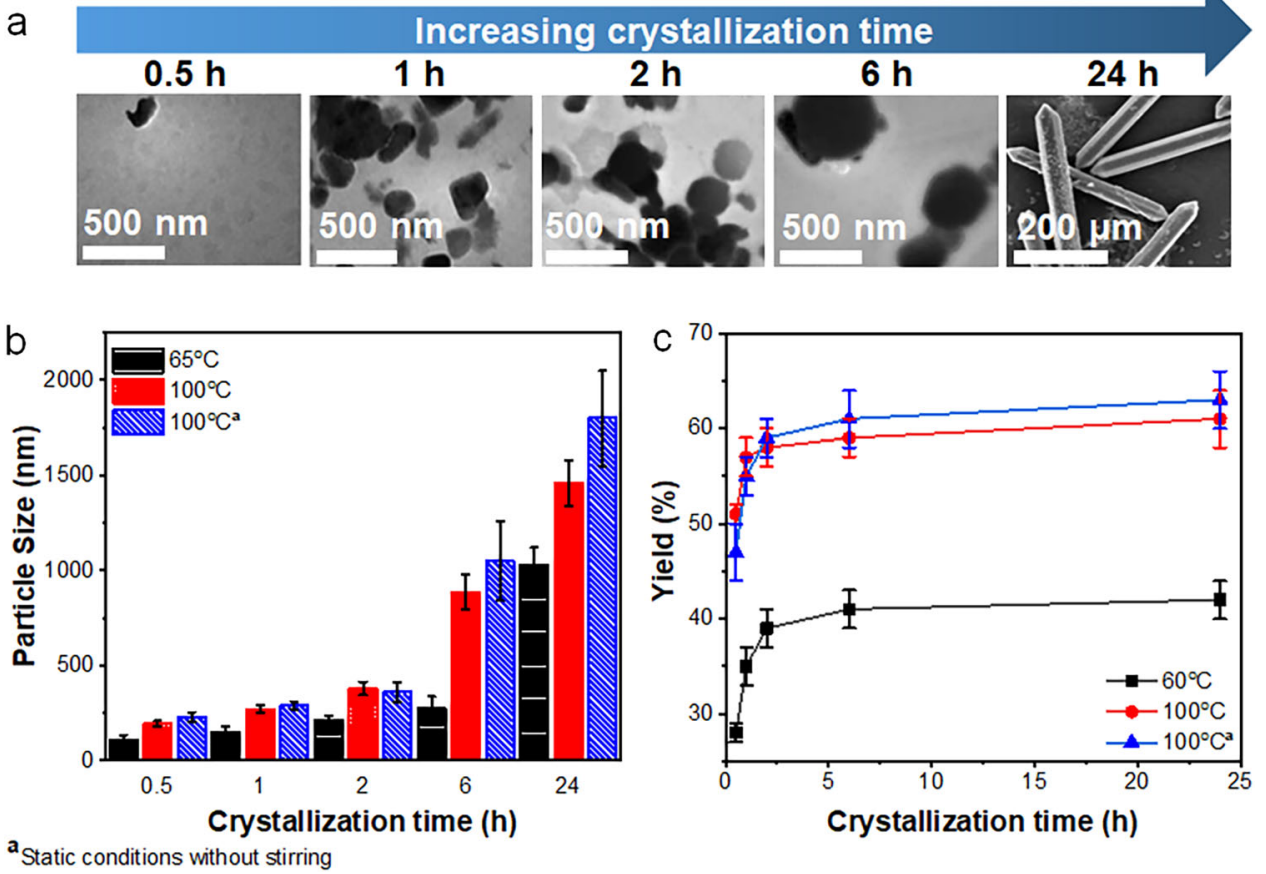

Fig. 2 Time and temperature dependence of MOFs downsizing. a TEM images of MIL-88A synthesized at $100^{\circ} \mathrm{C}$ for different reaction durations (SEM image for 24h). b Average particle size and $\mathbf{c}$ percent yield of MIL-88 obtained for varying reaction temperatures and durations at ambient pressure. Adapted with permission from ref. ${ }^{39}$. Copyright @ 2010 The Royal Society of Chemistry. 
effect of downsizing ZIF-67 MOFs as a potential oxygen reduction reaction (ORR) electrocatalyst was also studied, which revealed that the smallest $(300 \mathrm{~nm})$ nanocrystals showed the highest electrochemical performance. This dependence of the particle size on the electrochemical performance was observed through their respective electron transfer number $(n)$, where the $300 \mathrm{~nm}$ ZIF-67 acquired a value of 3.7 for a potential range from 0 to $0.7 \mathrm{~V}$. Large $800 \mathrm{~nm}$ crystals exhibited lower $n$ values with an average value of 3.5. A size enlargement to a $1.7 \mu \mathrm{m}$ decreased the $n$ value to 3.2 , and bulk crystals $(>10 \mu \mathrm{m})$ had the lowest $n$ value of 2.8 . However, shortened reaction durations may result in a decreased yield because metal and organic ligand precursors may remain unreacted in the solution ${ }^{46}$. An insufficient crystallization time may also result in poorly defined crystallites with inhomogeneous morphologies ${ }^{46}$. Therefore, methods with a reduced reaction time combined with techniques that lead to accelerated nucleation rates, such as varying the energy source, were also considered.

\section{Varying the energy source}

This section discusses alternative methodologies for solvothermal/hydrothermal synthesis of MOFs at the nanoscale. These methods, include microwave (MW) and ultrasonic (US) irradiation as well as mechanical and chemical driven approaches.
MW and US irradiation-assisted synthesis Heating via MW irradiation is an interesting approach for the synthesis of MOF nanoparticles. The main advantage of MW-assisted heating is its ability to apply concentrated and localized power to the precursor solution ${ }^{47-49}$. Energy is applied directly to the reaction solution rather than being conducted from the surface of the vessel. In addition, MW-assisted heating does not warm the air or the vessel, which essentially allows temperatures above the boiling point of the solvent with a minimal volume expansion within the vessel ${ }^{47,48,50}$.

Compared to conventional heating techniques, the MWassisted method permits shorter heating durations (the span of a few minutes) with a more concentrated power input, which results in increased nucleation (MOF seeds). Since the reaction time is decreased, this process only allows limited time for crystal growth and aggregation, thus resulting in a smaller particle size ${ }^{48,51}$. Through careful optimization of the irradiation power and duration, the MW-assisted method has led to the production of nanosized MOF crystals. For example, nanoporous nanosized Cr-MIL-101 crystals with sizes from 40 to $90 \mathrm{~nm}$ were prepared using MW-assisted method at varying energy inputs from 36 to $1440 \mathrm{~kJ}$ (from 1 to $40 \mathrm{~min}$ at $600 \mathrm{~W})^{27}$. It was found that the crystal size increased with MW irradiation time (Fig. 3a). Importantly, the crystallinity as well as the evolution of a refined shape and morphology (from spherical to cubic) increased with energy input (Fig. 3b).

a

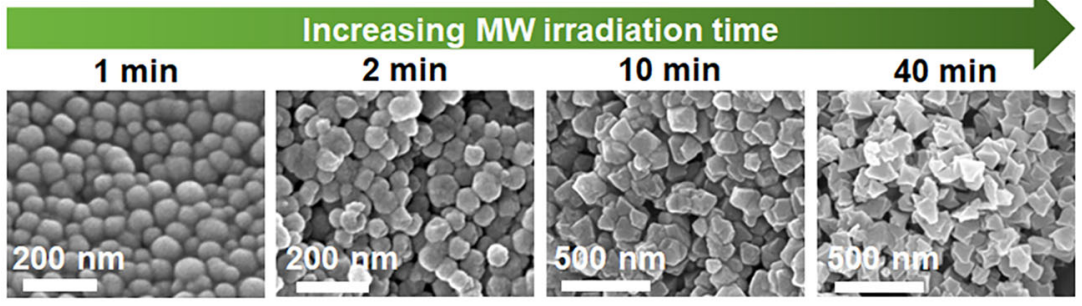

b

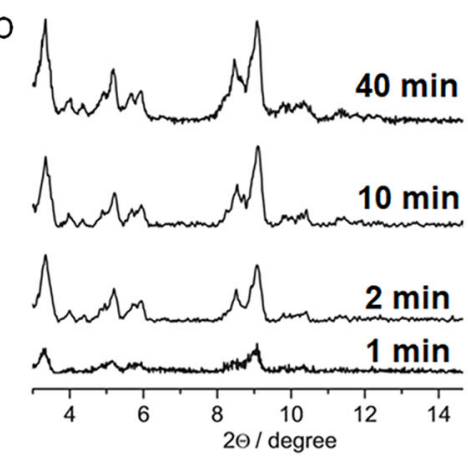

C

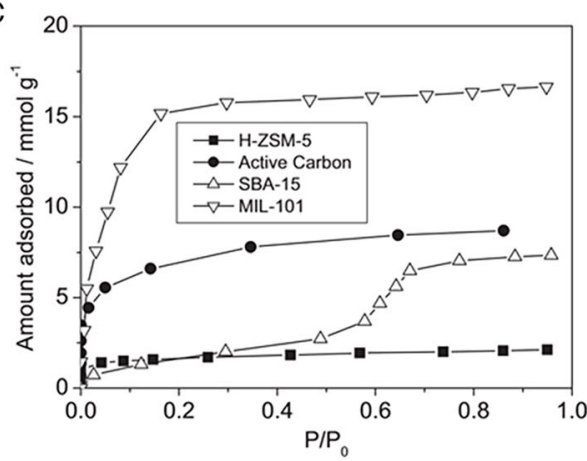

Fig. 3 Downsizing through efficient energy input. a Time-dependent change in the morphology of a MIL prepared with microwave irradiation at $210^{\circ} \mathrm{C}$ and $\mathbf{b}$ the corresponding XRD profile. $\mathbf{c}$ Benzene adsorption of nanosized MIL-101 compared to that of other nanoporous materials. Adapted with permission from ref. ${ }^{27}$. Copyright $\odot 2007$ WILEY-VCH Verlag GmbH \& Co., KGaA, Weinheim. 
Interestingly, this technique only took $40 \mathrm{~min}$ at $210{ }^{\circ} \mathrm{C}$ to achieve homogeneous cubic MIL-101 crystals (Fig. 3a), which is significantly shorter than the conventional hydrothermal method that takes $10 \mathrm{~h}$ at $220^{\circ} \mathrm{C}$. The particle sizes acquired through the $\mathrm{MW}$-assisted method ranging from 40 to $90 \mathrm{~nm}$ are also smaller than the typical size from 200 to $400 \mathrm{~nm}$ that is produced via traditional solvothermal methods. The adsorption capacity of MWprepared MIL-101 nanocrystals for benzene measured at $30{ }^{\circ} \mathrm{C}$ was estimated to be $16.7 \mathrm{mmol} \mathrm{g}^{-1}$ at $P P_{0}^{-1}=0.5$. This value is higher than that for other commercially available mesoporous materials, such as silica (SBA-15), zeolite (HZSM-5), and activated carbon, which have adsorption capacities of $3.0,1.9$, and $8.0 \mathrm{mmol} \mathrm{g}^{-1}$, respectively, when measured at the same temperature and $P P_{0}^{-1}$ conditions (Fig. $\left.3 \mathrm{c}\right)^{27}$.

Other reported $\mathrm{MW}$-assisted downsizing strategies of MOFs include the miniaturization of HKUST-1 crystals to sizes ranging from 2 to $3 \mathrm{~nm}^{29}$ upon irradiation at $700 \mathrm{~W}$ for $4 \mathrm{~min}$ (168 KJ energy input). The resulting HKUST-1 nanocrystals also possessed a $\mathrm{SA}_{\mathrm{BET}}$ of $1,138 \mathrm{~m}^{2} \mathrm{~g}^{-1}$ and $\mathrm{N}_{2}$ adsorption of $15.9 \mathrm{mmolg}^{-1}$, which are comparable to those for HKUST-1 crystals synthesized solvothermally $\left(\mathrm{SA}_{\mathrm{BET}}\right.$ of $965 \mathrm{~m}^{2} \mathrm{~g}^{-1}$ and $\mathrm{N}_{2}$ adsorption of $13.8 \mathrm{mmol} \mathrm{g}^{-1}$ ). This study was also able to couple the technique with supercritical $\mathrm{CO}_{2}$ activation, which yielded crystals with a surface area that increased by $50 \%$ and resulted in an increased $\mathrm{N}_{2}$ uptake $\left(\mathrm{SA}_{\mathrm{BET}}\right.$ of $1587 \mathrm{~m}^{2} \mathrm{~g}^{-1}$ and $\mathrm{N}_{2}$ uptake of $21.4 \mathrm{mmol} \mathrm{g}^{-1}$ ).

Nanocrystals of isoreticular MOFs (IRMOF-1, -2 , and -3) were also synthesized by MW irradiation (150 W) within $25 \mathrm{~s}^{28}$. The crystal sizes were reduced to the submicrometer scale $(500 \mathrm{~nm}$ to $1 \mu \mathrm{m})$ from a bulk IRMOF crystal size of $4 \mu \mathrm{m}$ through the use of MW heating and dilute linker concentrations from 0.001 to $0.01 \mathrm{M}$. The edges and vertices of submicron-sized crystals, however, are less sharp than those of micronsized crystals, which is suspected to be a consequence of an insufficient time for crystal ripening due to a low energy input used during the synthesis process $(3.75 \mathrm{~kJ}$ for downsized crystals versus $9.0 \mathrm{~kJ}$ for bulk crystals).

Similar to the impact of MW irradiation, US irradiation is another unconventional technique that promotes enhanced crystallization kinetics, controlled particle morphology, and phase selectivity ${ }^{52}$. Similar to the behavior during MW irradiation, downsizing via US irradiation also starts with improved nucleation, which is primarily due to a localized high temperature and pressure brought about by the growth and collapse of generated acoustic cavitation ${ }^{52,53}$. The use of US-assisted synthesis to produce nanosized MOFs was first applied for nano-MOF $\left[\mathrm{Zn}_{3}(\mathrm{btc})_{2} \bullet 12 \mathrm{H}_{2} \mathrm{O}\right] \quad(\mathrm{btc}=1,3,5$-benzenetricarboxylic acid). Using this approach, crystals produced after $10 \mathrm{~min}$ of US irradiation $(40 \mathrm{kHz})$ with a power of
$60 \mathrm{~W}$ at room temperature showed sizes between 50 and $100 \mathrm{~nm}$ when observed by $\mathrm{TEM}^{30}$. This study also demonstrated a drastic increase in crystal sizes that ranged from 700 to $900 \mathrm{~nm}$ with an increased irradiation time of $90 \mathrm{~min}$. These results clearly demonstrate the morphological control of nanoscaled MOFs through a variation of the ultrasonication time.

MOF-177 [ $\left.\mathrm{Zn}_{4} \mathrm{O}(\mathrm{BTB})_{2}\right]$ microcrystals were synthesized with the aid of US irradiation ${ }^{31}$. MOF-177 crystals with sizes varying from 5 to $20 \mu \mathrm{m}$ were synthesized using a US-assisted approach and had a rapid reaction time of $40 \mathrm{~min}$ at $500 \mathrm{~W}$. The resulting crystals were $75-300$ times smaller than those produced by the solvothermal technique (crystal size $=1.5 \mathrm{~mm}$ ). The nanosized MOF177 had an improved $\mathrm{CO}_{2}$ capture performance with an adsorption capacity of $1315 \mathrm{mgg}^{-1}$ compared to the adsorption capacity of $1286 \mathrm{mg} \mathrm{g}^{-1}$ for millimeter-sized MOF-177. This study also showed significant downsizing of MOF-177 with a remarkably high yield of $95.6 \%$ compared to a yield of only $66.7 \%$ when the solvothermal technique was used. This report clearly demonstrated that US irradiation is an efficient technique for downsizing MOFs.

Since then, US-assisted methods have been widely utilized for the production of nano-MOFs ${ }^{6,31,54,55}$, including the production of HKUST-1 nanocrystals with an average particle size of $10 \mathrm{~nm}$ and an improved surface area and hydrogen storage capacity. HKUST-1 nanocrystals were formed at room temperature through ultrasonic exposure $(40 \mathrm{kHz})$ of the precursor solutions at various irradiation durations between 5 and $60 \mathrm{~min}$ at a fixed power of $60 \mathrm{~W}$. US irradiation of copper (II) acetate with $\mathrm{H}_{3} \mathrm{BTC}$ in a dimethylformamide/ethanol/water (DMF/ $\mathrm{EtOH} / \mathrm{H}_{2} \mathrm{O}$ at $\left.3: 1: 2, \mathrm{v} / \mathrm{v}\right)$ solution for $5 \mathrm{~min}$ provided a high yield of $62.6 \%$ (based on $\mathrm{Cu}$ content). Increasing the reaction duration to $60 \mathrm{~min}$ resulted in a substantial yield increase of up to $85.1 \%$, which is also higher than the yield for traditional hydrothermal or solvothermal HKUST-1 synthesis (up to $65 \%)^{6}$. Likewise, no obvious impurities in the samples were detected by XRD, which suggests that the HKUST-1 products were isostructural. The TEM results also verified that the powder consisted of uniform cubic crystals. Last, the specific surface area of the crystals acquired with this approach $\left(1100 \mathrm{~m}^{2} \mathrm{~g}^{-1}\right)$ was almost similar to the reported surface area for solvothermally produced crystals $\left(\sim 950-1200 \mathrm{~m}^{2} \mathrm{~g}^{-1}\right)^{6,29}$. The reported nanosized HKUST-1 crystals were also smaller in size than those acquired with the conventional solvothermal approach $(10-30 \mu \mathrm{m})$, which usually takes $10 \mathrm{~h}$ to conduct.

All of the aforementioned studies indicated that MWand US-assisted solvothermal methods significantly decreased the synthesis duration, which only took several minutes in most cases in contrast to that for classical 
solvothermal methods that usually takes several hours up to a few days. Both MW- and US-assisted methods offer effective, rapid, and facile synthesis options for MOFs that produce relatively high yields compared to those of traditional solvothermal techniques. However, it needs to be considered that different MOF systems have varying nucleation and crystal growth rates ${ }^{13}$. MW- and USassisted downsizing approaches may only be applicable for a limited number of MOFs and thus must be used in combination with other modification techniques to provide specific limits on the particle size. This combination includes tandem MW- and US-assisted synthesis with chemical methods, such as microemulsion and modulator-assisted methods. Moreover, the heating parameters, including the dielectric properties, power efficiency, penetration depth and power density, are essential factors for the scalability of MW and USassisted methods ${ }^{56}$. The current lack of information about these parameters is one key factor that hinders the scalability of MW- and US-assisted methods from the laboratory to an industrial scale.

Mechanochemical synthesis Another alternative technique is the use of mechanical grinding to promote the reaction and provide the necessary energy for the formation of MOFs. Mechanochemical synthesis is a solvent-free synthesis method for MOFs where chemical reactivity of a bulk reactant is achieved through the application of a mechanical force ${ }^{57}$. This technique offers a convenient green and scalable method to prepare microporous MOFs due to its minimal use of solvents. In addition, this technique provides readily downsized nano-MOFs due to the exposure of the material to physical grinding conditions, resulting in the production small fragments from large particles.

Solvent-free MOF synthesis was first applied in the production of microporous copper (II) isonicotinate MOF crystals $(<100 \mathrm{~nm}$ in size) that were obtained by grinding a mixture of copper (II) acetate and isonicotinic acid powders for $10 \mathrm{~min}$ without the application of additional heat ${ }^{57}$. The same approach was also used to synthesize commonly used MOFs, such as HKUST-1 ${ }^{58}$. The synthesis was carried out with conventional ball milling of the metal-salt precursors for $25 \mathrm{~min}$. The acquired HKUST-1 crystals were $50 \mathrm{~nm}$ in size, which is significantly smaller than their bulk counterparts (approximately 10-20 $\mu \mathrm{m}$ ) but with a comparable specific surface area $\left(1713 \mathrm{~m}^{2} \mathrm{~g}^{-1}\right)$ to that of the other HKUST-1 crystals reported earlier using different synthesis methods $\left(\sim 950-1200 \mathrm{~m}^{2} \mathrm{~g}^{-1}\right)^{6,29}$. Mechanochemical synthesis was also able to produce modified nano-MOFs that exhibited potential in new applications ${ }^{14}$. This approach produced monodispersed sulfur-modified ZIF-8 nanocrystals (S/ZIF-8) (100-200 nm) with a defined rhombic dodecahedral morphology (Fig. 4a, b)

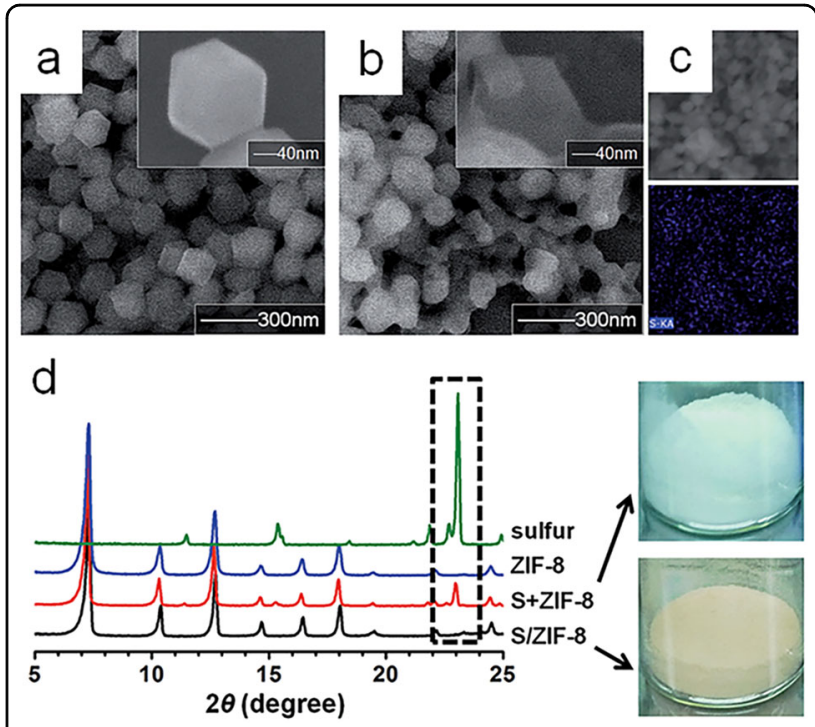

Fig. 4 Mechanochemical synthesis of ZIF-8 nanocrystals. SEM images of a blank ZIF-8 nanocrystals and $\mathbf{b}$ S/ZIF-8. c EDS map showing the sulfur distribution in S/ZIF-8. $\mathbf{d}$ XRD patterns of pure sulfur, ZIF-8 and S/ZIF-8 powders after grinding (S+ZIF-8) and after heating (S/ZIF-8). Adapted with permission from ref. ${ }^{14}$. Copyright (C) 2014 The Royal Society of Chemistry.

that possessed promising properties for sulfur storage. The samples also showed a uniform sulfur distribution, which was confirmed by EDS mapping (Fig. 4c), along with a wellmaintained crystalline ZIF-8 structure, which was confirmed by XRD (Fig. 4d).

With the optimal combination of electrolyte and cut-off voltage range, the sulfur stored in an appropriate MOF host can be used for intercalation (fast and stable) and conversion (high energy density) cathodes ${ }^{14}$. The S/ZIF-8 nanocrystal with $30 \mathrm{wt} \%$ sulfur loading achieved a better discharge capacity of $1055 \mathrm{~mA} \mathrm{hg}^{-1}$ at $0.1 \mathrm{C}$ than micrometer-sized ZIF- 8 crystals that had a discharge capacity of only $556 \mathrm{~mA} \mathrm{hg}^{-1}$. The $0.08 \%$ decay per cycle over 300 cycles at $0.5 \mathrm{C}$ exhibited by the samples is also important for long-cycle life $\mathrm{Li}-\mathrm{S}$ batteries ${ }^{14}$. These findings illustrate that the S/ZIF-8 nanocrystals are plausible candidates as new hosts for sulfur for the production of effective and stable $\mathrm{Li}-\mathrm{S}$ batteries.

Though relatively new and unusual, grinding a mixture of metal salts and organic ligands could serve as a simple, convenient, and effective preparation method for nanosized MOFs. The process is quick and high-yielding, and its solvent-free nature could enable its ability to be scaled up in terms of the materials used and energy/time efficiency compared to those of the traditional solvothermal approach. The technique also avoids several drawbacks of solvent-based MOF synthesis methods, such as the entrapment of solvents within a channel, which 
requires an additional activation step that often leads to a collapse of the framework ${ }^{59,60}$.

However, assuring the completeness of the reaction, maintaining the defined pore metrics (size, shape, and distribution) of the original MOF and ensuring consistent application of a mechanical force throughout the reaction mixture are critical points to be considered when using this approach ${ }^{59,61}$. Similarly, consistency in the size and shape of the acquired crystal is highly variable in terms of the strength and duration of grinding ${ }^{62}$, which leads to reproducibility issues for this technique.

\section{Chemical growth modifications}

Despite being able to produce nanocrystals through the modification of synthesis variables, achieving complete crystallization with precise morphological control remains a challenge. Because of this difficulty, methods that provide sophisticated shape control and reaction zone confinement have been explored. Typical methods for the preparation of MOFs, including solvothermal/hydrothermal synthesis with a modified solvent environment ${ }^{32}$ and the presence of additives, such as emulsifiers ${ }^{40}$, surfactants ${ }^{40}$, and stabilizing polymers ${ }^{63}$, are each discussed in the succeeding section.

\section{Solvation effects}

Various procedures have been used to tune both the size and morphology of MOFs, but the modification of the solvent composition is one of the most convenient methods. Conventional solvents for MOF formation include $\mathrm{N}, \mathrm{N}$-dimethylformamide, $\mathrm{N}, \mathrm{N}$ - diethylformamide, dimethyl sulfoxide, and $\mathrm{N}$-methylpyrrolidone. However, the use of mixed solvents with water or ethanol (e.g., $\mathrm{DMF} /$ ethanol and DMF/water) was found to influence crystal growth through solvent-induced effects ${ }^{32,64}$. These synthesis methods revealed that tuning the hydrogen bonding interaction between the solvent and the ligand affects the formation mechanism of MOFs, leading to a precise control of the size and shape $\mathrm{e}^{33}$. These routes also provided new insights into solvation effects, which are especially useful for the controlled and optimized crystallization of MOFs in aqueous solvents.

The formation of HKUST-1 in water/ethanol solvent systems at ethanol volume ratios greater than $30 \mathrm{vol} \%$ yielded crystals with sizes ranging from 20 to $300 \mathrm{~nm}^{32}$. This size range is significantly smaller than that of bulk HKUST-1 crystals that have a typical size distribution range from 10 to $30 \mu \mathrm{m}$. In this setup, the addition of excess ethanol to the solution $(>30 \mathrm{vol} \%)$ induced the formation of water clusters that were surrounded by ethanol, which reduced the interaction of the water with the linker (Fig. 4a). The minimized interaction of the $\mathrm{Cu}^{2+}$ ions with water molecules effectively enhanced their coordination with carboxylate groups $\left(\mathrm{H}_{3} \mathrm{BTC}^{+}\right)$, which led to favorable nucleation and promoted the formation of small MOF nanocrystals (Scheme in Fig. 5a, SEM images in Fig. 5b).

Increasing the ethanol content also resulted in welldefined XRD profiles that are consistent with the simulated HKUST-1 pattern. This finding implies that the presence of water prevented the coordination between carboxyl groups and $\mathrm{Cu}^{2+}$ ions, which resulted in premature HKUST-1 crystals. The $\mathrm{SA}_{\mathrm{BET}}$ values of the samples synthesized at low ethanol content were also extremely low (ranging from 8 to $11 \mathrm{~m}^{2} \mathrm{~g}^{-1}$ ) compared to crystals acquired at a high ethanol content $\left(1067 \mathrm{~m}^{2} \mathrm{~g}^{-1}\right)$. This decrease in the $\mathrm{SA}_{\mathrm{BET}}$ was attributed to the collapsed HKUST-1 crystal structures that caused a drastic decrease in the porosity. This result agrees with the XRD data that showed that crystals did not form in water/ethanol mixtures of less than $33 \mathrm{vol} \%$ ethanol.

$\mathrm{NH}_{2}$-MIL-53(Al) crystals synthesized in a DMF/water mixed solvent system also yielded nanocrystals with varying sizes and shapes that depended on the solvent composition $^{33}$. A small amount of water (3.3 vol\%) facilitated the deprotonation of the carboxylate group in the organic linker $\left(\mathrm{NH}_{2}-\mathrm{BDC}\right)$. However, excess water $(>50 \mathrm{vol} \%)$ inhibited the deprotonation and solubility of the ligands. A low water content in the mixture resulted in $1.65 \mu \mathrm{m}$ aggregates of nanosized $\mathrm{NH}_{2}-\mathrm{MIL}-53(\mathrm{Al})$ (approximately $24 \mathrm{~nm}$ in diameter per crystal), while having excess water resulted in crystal sizes of up to $>500 \mathrm{~nm}$ per particle. Having an optimum water content of $25 \mathrm{vol} \%$ resulted in $76 \mathrm{~nm}$ crystals. Furthermore, the largest $\mathrm{SA}_{\mathrm{BET}}$ of these samples $\left(1882 \mathrm{~m}^{2} \mathrm{~g}^{-1}\right)$ is nearly twice as large as the reported $\mathrm{SA}_{\mathrm{BET}}$ for large MIL-53 MOFs $\left(994 \mathrm{~m}^{2} \mathrm{~g}^{-1}\right.$, sizes $\left.>500 \mathrm{~nm}\right)$.

Co-MOF-74 crystals were also precisely synthesized and uniformly downsized to nanorods with dimensions of $20 \mathrm{~nm}$ (diameter) and $240 \mathrm{~nm}$ (length) using a 1:4 DMF/ water mixture ${ }^{18}$. Aside from the high surface area $\left(874 \mathrm{~m}^{2} \mathrm{~g}^{-1}\right)$, the downsized crystals also demonstrated a high $\mathrm{O}_{2}$ adsorption $\left(9.6 \mathrm{~cm}^{3} \mathrm{~g}^{-1}\right)$ and discharge capacity $\left(11,350 \mathrm{mAhg}^{-1}\right)$ compared to those of their bulk counterparts $\left(\mathrm{SA}_{\mathrm{BET}}=669 \mathrm{~m}^{2} \mathrm{~g}^{-1}, \mathrm{O}_{2}\right.$ adsorption $=$ $3.1 \mathrm{~cm}^{3} \mathrm{~g}^{-1}$ and discharge capacity $\left.=11,350 \mathrm{~mA} \mathrm{~h} \mathrm{~g}^{-1}\right)^{18}$. This improvement in the $\mathrm{O}_{2}$ uptake and discharge capacity makes nanosized Co-MOF-74 suitable candidates as cathode materials for high-capacity $\mathrm{Li}-\mathrm{O}_{2}$ batteries.

In summary, a judicious solvent choice showed promising potential for nano-MOF synthesis. Unlike previously discussed additive-free methods, this approach resulted in complete reactions since the precursors were homogeneously dissolved in the reaction solution and crystallized completely. Importantly, the resulting products contained a small amount of impurities since no additives were introduced to the reaction. However, the 


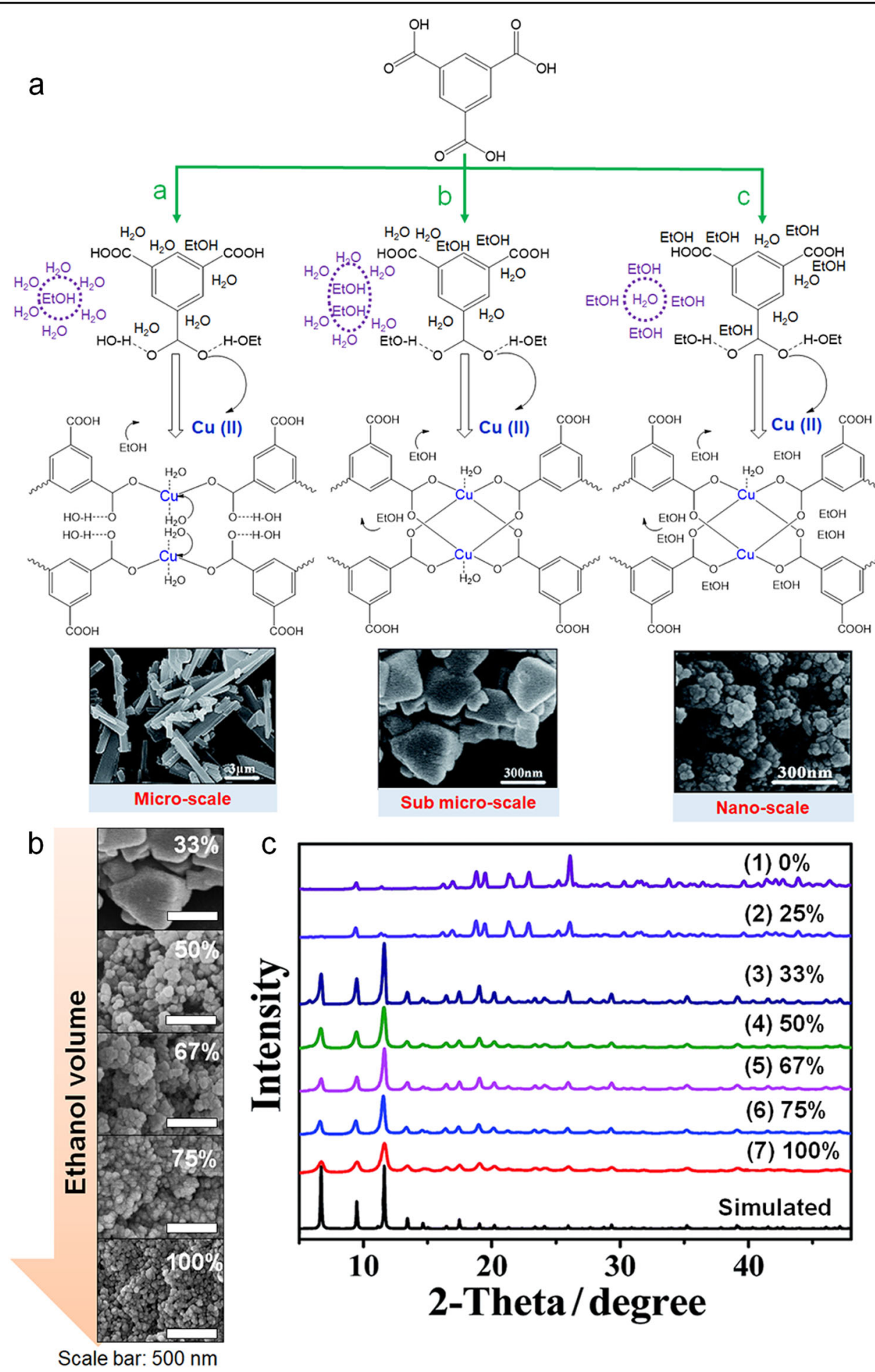

Fig. 5 Solvation-controlled MOFs crystallization. a Formation mechanism of HKUST-1 and the effect of water/ethanol percentage on the particle size. $\mathbf{b}$ SEM images of HKUST-1 synthesized in water/ethanol mixtures with ethanol volume ratios. c XRD patterns of HKUST-1 prepared in varying water/ethanol percentages. Adapted with permission from ref. ${ }^{32}$. Copyright $\odot 2015$ The Royal Society of Chemistry. 
appropriate solvent ratios, which provided controlled metal-ligand interactions, varied for different types of MOFs. In this regard, tuning of the parameters, such as the solubility of the precursors and deprotonation rate of the organic linker in the solvent system, must be done to use this approach.

\section{Addition of surfactants during MOF crystallization}

Surfactants are known surface stabilizers and templates for synthesizing various nanoparticles (NPs). The binding of NPs to a surfactant results in a decreased surface energy, precise morphological control, and stable colloidal dispersion. In the case of MOFs, surfactants that act as capping molecules in the crystallization process limit aggregation through reaction confinement in the surfactant micelles. As a result, the crystal growth and morphology are limited by the size and shape of the dispersed phase, and the surfactant coating provides stability against agglomeration ${ }^{19,65}$.

There have been reports on the downsizing of porous inorganic materials through surfactant-assisted synthesis ${ }^{65}$. A commonly used strategy involves cetyltrimethylammonium bromide (CTAB) stabilization. A perfect example of size control using $\mathrm{CTAB}$ was successfully demonstrated through the miniaturization of isoreticular MOFs (IRMOFs) ${ }^{41}$. Octahedral IRMOF-1 (MOF-5) and tetragonal IRMOF-3 crystals with sizes of approximately 200 and $300 \mathrm{~nm}$ were acquired via CTAB-assisted solvothermal synthesis; this size is 20 times smaller than the original IRMOF dimension of approximately $4 \mu \mathrm{m}$. These crystals were successfully produced with equally high yields of up to $99 \%$ (stoichiometric yield based on organic ligands). However, an additional heating step at $300^{\circ} \mathrm{C}$ was required to utilize the optimum capacity of the products since excess surfactants may have resulted in pore clogging, which is shown by their low $\mathrm{SA}_{\mathrm{BET}}$ of $\sim 400 \mathrm{~m}^{2} \mathrm{~g}^{-1}$ (SABET upon activation $>3000 \mathrm{~m}^{2} \mathrm{~g}^{-1}$ ).

In addition to a size reduction, precise morphology control was demonstrated in another study that used ambient temperature synthesis of MOF- $5^{40}$ (Fig. 6). The surfactant-assisted synthesis of MOF-5 resulted in rodshaped nanocrystals with lengths of $200-500 \mathrm{~nm}$, widths of $50-100 \mathrm{~nm}$, and thicknesses of $50-80 \mathrm{~nm}^{40}$ when the MOF-5 had an original irregular morphology with a size range from 1 to $2 \mu \mathrm{m}$ (without adding CTAB).

The downsized crystals also demonstrated excellent nitroaromatic explosive sensing properties compared to that of their bulk counterparts. The adsorption of nitrobenzene by the MOFs, observed through fluorescence quenching (excitation and emission at 304 and $427 \mathrm{~nm}$ ), took $250 \mathrm{~s}$ for the nanocrystals compared to $1500 \mathrm{~s}$ for the microcrystals. This observation indicates faster adsorption of nitrobenzene by MOF- 5 due to the larger exposed surface area of the nanocrystals compared with that of the microcrystals.

Surfactants other than CTAB could also be used to minimize the growth of MOF crystals. ZIF-8 nanocrystals (average diameter of $57 \mathrm{~nm}$ and thickness of $42 \mathrm{~nm}$ ) were acquired through $24 \mathrm{~h}$ ambient temperature synthesis in the presence of poly(diallyldimethylammonium chloride) (average MW 400,000 to $500,000 \mathrm{Da})^{42}$. The presence of surfactants during room temperature synthesis had no significant effect on the rhombic dodecahedral morphology of ZIF-8, which retained a relatively similar $\mathrm{SA}_{\mathrm{BET}}$ $\left(1264 \mathrm{~m}^{2} \mathrm{~g}^{-1}\right)$ compared to that for ZIF-8 acquired through other methods $\left(\sim 1000 \mathrm{~m}^{2} \mathrm{~g}^{-1}\right)^{42,43}$.

ZIF-8 with a size range from 34 to $59 \mathrm{~nm}$ and a surface area of $1599 \mathrm{~m}^{2} \mathrm{~g}^{-1}$ were also obtained through the MWassisted method (1000 W for $30 \mathrm{~min}$ ) in the presence of
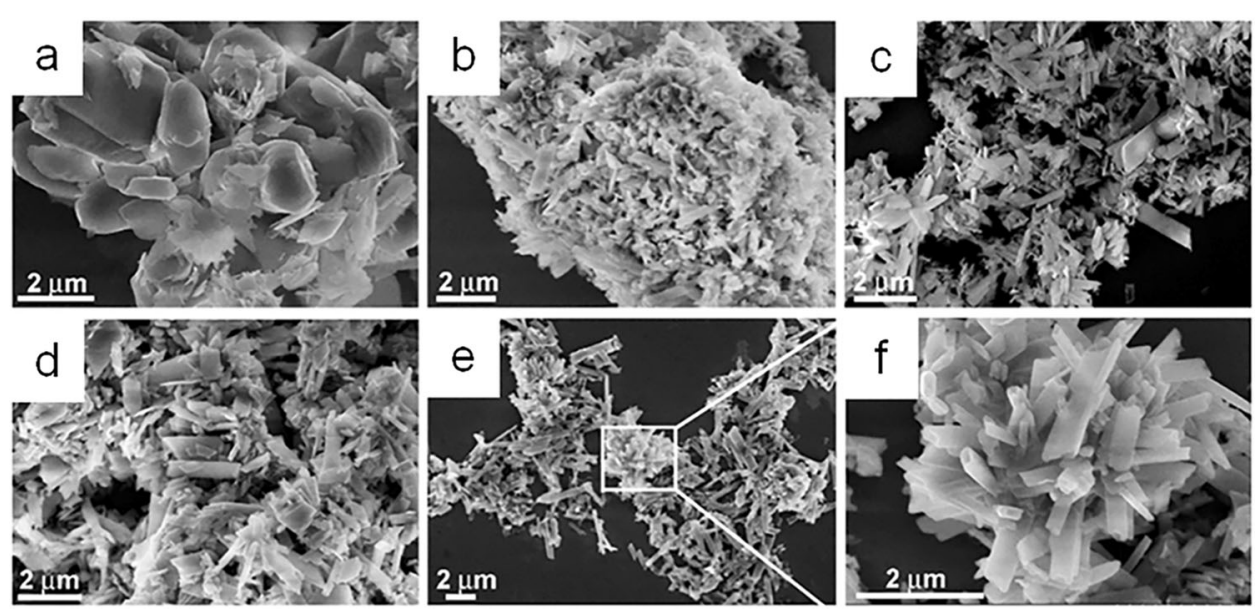

Fig. 6 Limiting MOFs crystal growth using surfactants. SEM images of a surfactant-free synthesized MOF- 5 with a 360 min crystallization time and CTAB-assisted synthesis at varying synthesis durations: b $10 \mathrm{~min}, \mathbf{c} 30 \mathrm{~min}, \mathbf{d} 180 \mathrm{~min}$, and $\mathbf{e}, \mathbf{f} 360 \mathrm{~min}$, where $\mathbf{f}$ is the magnified section of (e). Adapted with permission from ref. ${ }^{40}$. Copyright @ 2011 Elsevier B.V. All rights reserved. 
Pluronic P123. This method resulted in crystals approximately 10 times smaller than those acquired through the same method without a surfactant $(0.2$ to $0.4 \mu \mathrm{m}$ in size with a $\mathrm{SA}_{\mathrm{BET}}$ of $\left.\sim 1000 \mathrm{~m}^{2} \mathrm{~g}^{-1}\right)^{43}$. This result clearly shows that surfactant-assisted synthesis can work with different energy sources for crystallization.

The versatility of the surfactant-assisted method has led to the production of nanosized MOFs that both exhibit high monodispersity and colloidal stability. This feature is extremely attractive for uptake and release kinetics studies in liquids and is ideal for catalytic studies and biomedical applications ${ }^{66}$. However, the scalability of this approach is limited by its complex postsynthetic purification process to remove the surfactant from the final products.

\section{lonic-liquid microemulsions}

The use of ionic-liquid microemulsions (ILMEs) is a relatively new technique for tuning the size distributions of MOFs. In this method, the MOF building blocks are first dissolved in the aqueous phase and then dispersed as nanodroplets in an IL before being subjected to solvothermal conditions ${ }^{44}$ (scheme presented in Fig. 7a). ILs were previously used as an alternative solvent for zeolite synthesis, and their use in the synthesis of MOFs offers an environmentally friendly solvent system primarily due to their ease of recovery. ILs also possess several outstanding properties, such as high thermal stability, exceptional dissolution performance, versatility in choosing the cation and anion combination, and low vapor pressure ${ }^{67}$. In addition, the presence of cation and anion groups in the solvent can serve as charge compensating groups during synthesis ${ }^{62}$.

The use of IL solvents for MOF synthesis was first explored using [1-butyl- 3-methylimidazolium] $\left[\mathrm{BF}_{4}\right]$ $\left(\mathrm{BmimBF}_{4}\right)$ for the synthesis of $[\mathrm{Cu}(\mathrm{I})(\mathrm{bpp})]\left[\mathrm{BF}_{4}\right] \mathrm{MOFs}^{62}$. The effectiveness of the ILME technique for particle growth control, however, depends on the dispersion of the ligands in the aqueous phase. As demonstrated in the synthesis of ZIFs in [1-butyl- 3-methylimidazolium] $\left[\mathrm{PF}_{6}\right]$ $\left(\mathrm{BmimPF}_{6}\right)$ when a water-soluble organic linker was used,

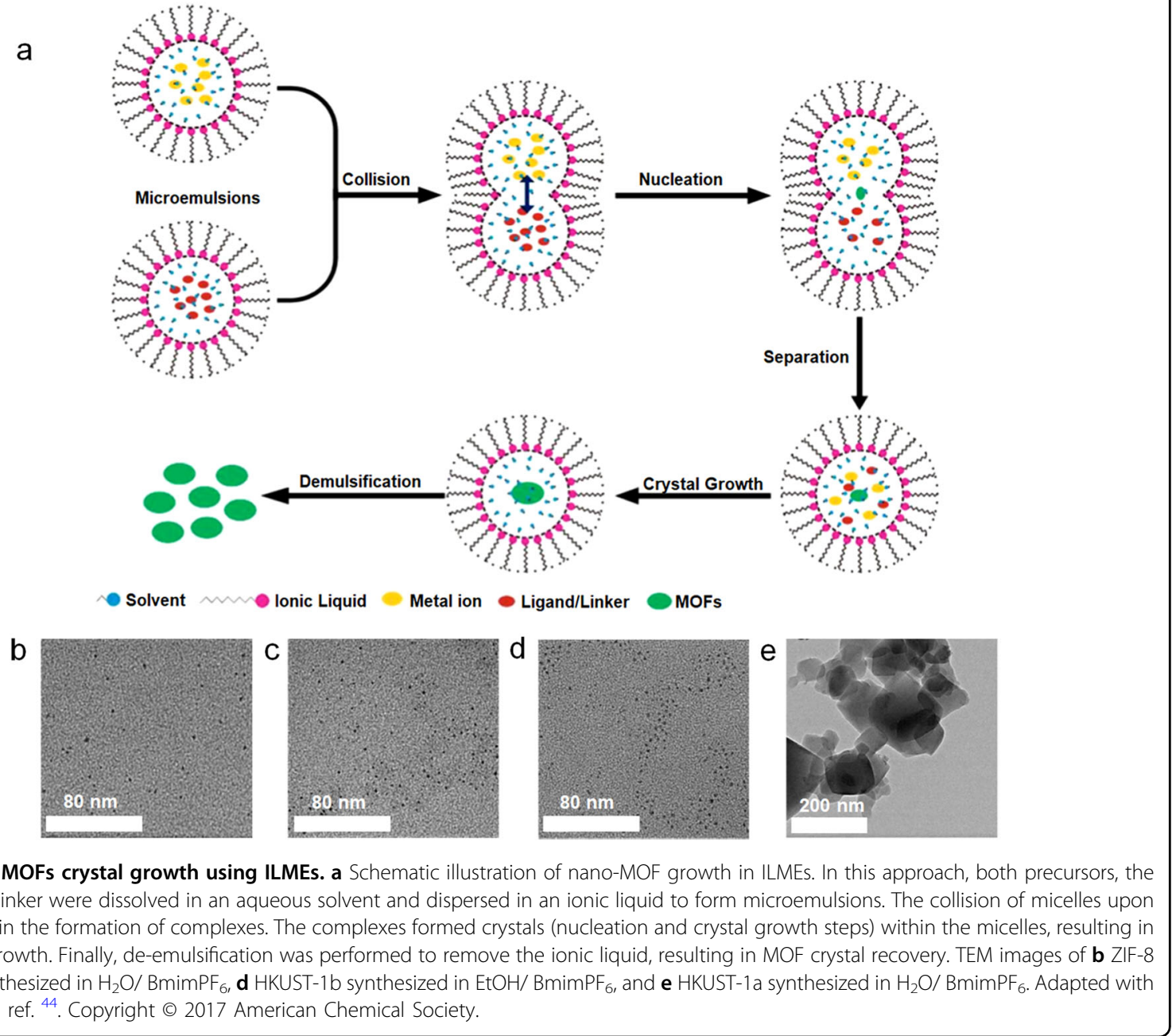


namely, (2-methylimidazole), the coordination reaction proceeded within the water nanodroplets to produce nanoscale ZIF-8 and ZIF-67 with sizes between 2.2 and $2.3 \mathrm{~nm}^{44}$. However, most MOFs are synthesized with water-insoluble organic linkers, such as 1,3,5-benzenetricarboxylic acid (BTC), necessitating a new method to improve the solubility of these ligands in the droplets of ILMEs. The addition of EtOH to the microemulsion mixture enables the dissolution of both $\mathrm{Cu}^{2+}$ and BTC in a modified nanodroplet of $\mathrm{H}_{2} \mathrm{O} / \mathrm{EtOH}$ and enables the formation of HKUST-1 crystals with a mean particle size of $1.6 \mathrm{~nm}^{44}$ (Fig. 7). Particle sizes of 1.6, 2.2, and $2.3 \mathrm{~nm}$ were obtained for HKUST-1, ZIF-8, and ZIF-67, respectively, using this technique and were almost as small as their respective crystallographic unit cells. TEM studies of bulk HKUST-1, ZIF-8, and ZIF-67 reported unit cell sizes of $2.6,1.48$, and $1.7 \mathrm{~nm}$, respectively ${ }^{52}$.

MOF-5 nanospheres were also synthesized in a combined IL/surfactant emulsion system. This approach used IL 1,1,3,3-tetramethylguanidinium acetate/surfactant Nethyl perfluorooctylsulfonamide (N-EtFOSA) charged with supercritical $\mathrm{CO}_{2}$ that was maintained at $16.8 \mathrm{MPa}$ and $80^{\circ} \mathrm{C}$ and accompanied by vigorous stirring ${ }^{45}$. Monodispersed nanospheres of approximately $80 \mathrm{~nm}$ in diameter were formed, which is 2.5 times smaller than that from CTAB-modified synthesis ${ }^{40}$ and 50 times smaller than bulk crystals ${ }^{40}$.

The unprecedentedly small individual particle size of the MOFs highlights the promising potential of this technique. However, these studies have only demonstrated the effect of ILMEs on the size of MOFs. Further studies are necessary to investigate the MOF crystallinity and adsorption properties of nanosized MOFs.

\section{Use of coordination modulators}

Coordination modulators are polymer molecules that typically act as monodentate and/or bidentate ligands and facilitate nucleation but subsequently decrease the rate of crystal growth, resulting in downsizing ${ }^{34,59,63}$. Commonly used modulators include polymers containing carboxylate, formate, acetate, and/or imidazolate functionalities that increase the $\mathrm{pH}$ of solutions ${ }^{34,60}$. The presence of a modulator induces the deprotonation of organic linker ions, which has preferable coordination with metal ions $^{34,60}$. This deprotonation results in increased nucleation rates and decreased crystal sizes due to the abrupt lowering of precursor supersaturation ${ }^{34,60}$. The nanocrystals are also stabilized with carboxylate groups from the modulator, which then serve as capping agents to prevent further crystal growth.

The addition of dodecanoic acid to the precursor solution of HKUST-1 effectively resulted in nanosized crystals using either solvothermal or MW-assisted methodologies $^{35}$. This study also established the effect of ligand and modulator compositions on the shapes and sizes of the acquired crystals (Fig. 8). As summarized in Fig. 8a, it is also important to carry out the synthesis with a minimum amount of modulator and a fast nucleation time to achieve the smallest crystal sizes. Crystals varying from $20 \mathrm{~nm}$ spheres up to $2 \mu \mathrm{m}$ cubes were achieved depending on the ligand and modulator concentration. It was also confirmed that products have a high porosity $\left(\mathrm{SA}_{\mathrm{BET}}\right.$ of $\left.1270 \mathrm{~m}^{2} \mathrm{~g}^{-1}\right)$ that is comparable to that of bulk samples $\left(\sim 950-1200 \mathrm{~m}^{2} \mathrm{~g}^{-1}\right)$.

Similarly, HKUST-1 crystals were also downsized through coordination modulation by using poly(acrylic acid) (PAA) as a stabilizing agent ${ }^{63}$. Downsizing from an original size of $10-20 \mu \mathrm{m}$ to less than $100 \mathrm{~nm}$ with preserved crystal shapes and facets was achieved by adding PAA as a capping agent. The improved surface area for the nanocrystals led to enhanced liquid phase oxidation of dibenzylamines into imines with a conversion rate of $53 \%$ compared to the $17 \%$ rate for the bulk samples. In another study, benzoic acid-modulated synthesis of $\mathrm{Zr}$-based MOFs (from the $\mathrm{UiO}$ family) resulted in crystal sizes from $230 \mathrm{~nm}$ to $1 \mu \mathrm{m}$, which is smaller than that for the UiO family $(>2 \mu \mathrm{m})^{36}$. However, the crystals changed from octahedral to irregular and spherical in shape and the $\mathrm{SA}_{\mathrm{BET}}$ decreased from $1400 \mathrm{~m}^{2} \mathrm{~g}^{-1}$ to $600 \mathrm{~m}^{2} \mathrm{~g}^{-1}$ for the bulk and downsized samples, respectively.

ZIF-8 crystals with sizes ranging from $10 \mathrm{~nm}$ and $1 \mu \mathrm{m}$ were also achieved through the use of $n$-heterocyclic and alkyl amines as modulators ${ }^{34}$. The synthetic strategy in this study also provided insights into the kinetics of the downsizing process, where auxiliary modulating ligands acted as competitive ligands for metal-linker coordination. The experiments also revealed that nanocrystal formation occurs when there is continuous slow nucleation followed by a short and quick crystal growth stage. Another interesting highlight of this study is the remarkable redispersibility and stability of the resulting nano-MOFs in organic solvents, which makes the approach attractive for a wide range of applications.

In a similar study, the role of various carboxylic acid modulators ( $\mathrm{R}-\mathrm{COOH}$, where $\mathrm{R}=\mathrm{H}, \mathrm{CH}_{3}, \mathrm{CF}_{3}$, and $\mathrm{CHCl}_{2}$ ) on the growth of UiO-66 MOFs and their effects on the surface properties and colloidal stability of the downsized crystals were further assessed ${ }^{37}$. It was emphasized that a variation in the identity of the carboxylic acid group through different hydrocarbon functionalities led to a controlled size distribution between $20 \mathrm{~nm}$ and $1 \mu \mathrm{m}$. The $\mathrm{pH}$ of the solution, which was achieved through a variation of the carboxylic acid concentration, also played a significant role in governing the morphology of the formed crystals. A high $\mathrm{pH}$ led to quasi-spherical crystals, while a low $\mathrm{pH}$ yielded octahedral crystals. 


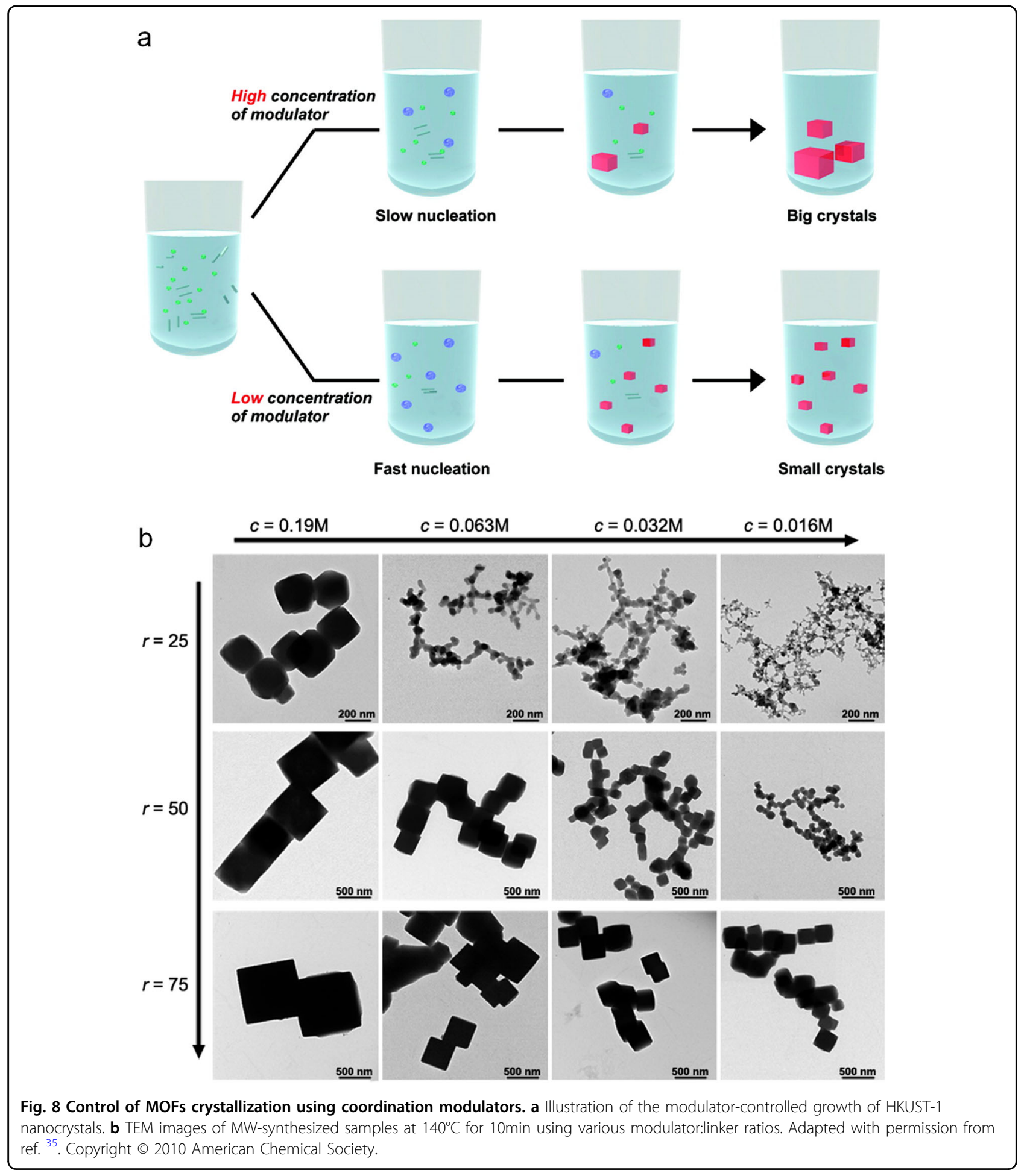

The vast use of coordination modulators for MOF synthesis has also led to the production of stable and redispersed nanoparticles with promising potential in biomedicine, material assembly, and catalysis. Although redispersible MOF nanoparticles could also be achieved through surface functionalization (with polymers and surfactants) ${ }^{37}$, surfactant molecules tend to clog solventaccessible pores/channels, leading to a decreased specific surface area and loading capacity ${ }^{68}$. Therefore, simpler methods, such as synthetic modulation, that could achieve colloidal stability with preserved pore accessibility, are being evaluated. However, similar to that for surfactant 
addition and ILMEs, the selection of organic polymers and ideal synthesis conditions may still require further optimization. In this case, a certain approach might be optimal for a specific MOF but may not be applicable for another system (i.e., amine modulation vs. carboxylic acid modulation), and the approach depends on certain factors, such as metal-ligand interactions, linker deprotonation and solvation effects.

\section{Top-down methods}

As discussed in the previous sections, methods for preparing nanosized MOFs in the presence of additives such as surfactants ${ }^{40}$ and stabilizing polymers ${ }^{63}$ are only applicable to a limited number of MOF materials. They also require a large quantity of organic solvents that, if not totally removed, make the MOFs unsuitable for biomedical applications. In addition, the grafted organic molecules also decrease the adsorption/loading capacity and accessibility of the framework to ions, gases and/or drug molecules ${ }^{61}$.

With these considerations, methods that do not require many chemical modifications are necessary ${ }^{33}$. The most recent fabrication method for nano-MOFs constitutes a "top-down" or "deconstruction" of bulk MOF materials ${ }^{69}$. This top-down approach has gained significant attention for downsizing bulk crystals due to its scalability. This section highlights the top-down size-reduction methods that produced nanocrystals by the exfoliation of MOF crystals through ultrasonication, ball milling, and grinding without additives.

\section{Mechanical downsizing-manual grinding and ball milling}

In contrast to the properties of chemical downsizing methods, mechanical grinding is the simplest and safest alternative to produce nanosized MOF crystals. This method is typically performed by physically fragmenting bulk MOF crystals through grinding techniques, such as solid-state ball milling and manual grinding.

One of the first demonstrations of mechanical grinding as an alternative downsizing route was utilized in the synthesis of gadolinium (III)- 1,4-bis(5-carboxy-1H-benzimidazole-2yl)benzene) (Gd-pDBI) nanocrystals for intravenous injection applications ${ }^{70}$. Bulk millimetersized Gd-pDBI $(0.5 \mathrm{~mm})$ that was grown in $\mathrm{H}_{2} \mathrm{O} / \mathrm{DMF}$ at $110^{\circ} \mathrm{C}$ for $72 \mathrm{~h}$ were reduced to $120 \mathrm{~nm}$-sized rodshaped nanocrystals with a particle size of $140 \mathrm{~nm}$ (Fig. 9).

Interestingly, the resulting nanosized MG-Gd-pDBI exhibited structural integrity, good crystallinity, and high porosity. The dispersions of the downsized crystals were also stable in a variety of solvents, such as water, ethanol, and DMF, for more than 90 min after sonication (Fig. 9b). The nanosized MG-Gd-pDBI also exhibited low blood toxicity and a high adsorption of an anticancer drug

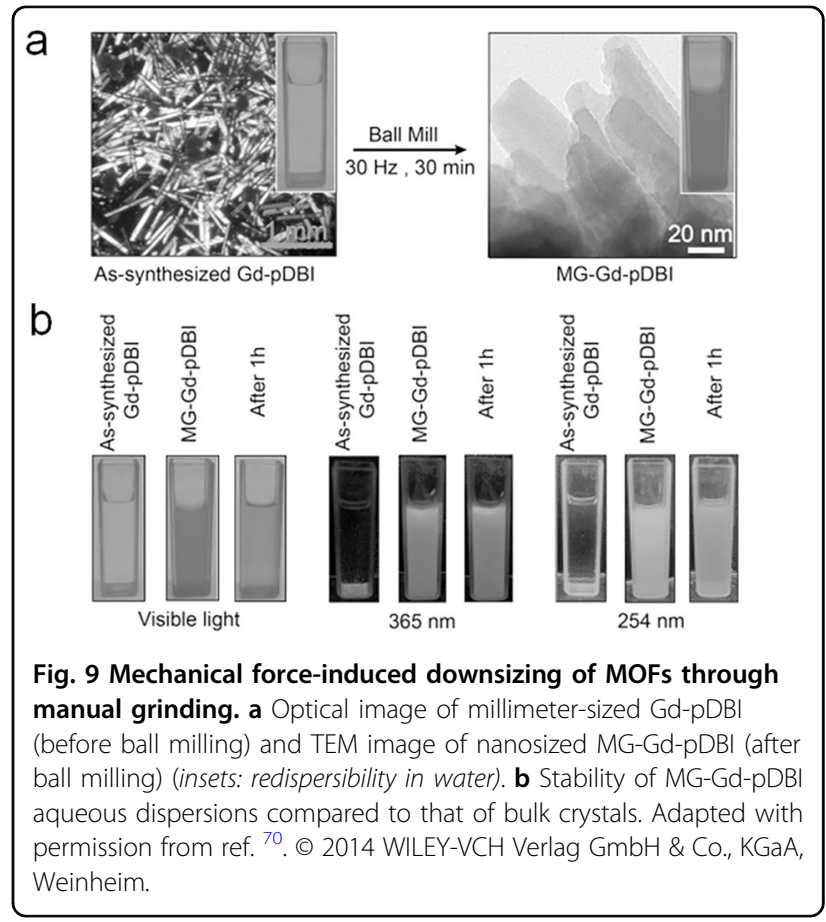

(doxorubicin) of up to $12 \mathrm{wt} \%$. The amount of adsorbed doxorubicin in this material is the highest reported to date compared to those absorbed by $\mathrm{pH}$-responsive and cancer cell-specific drug carriers.

In another study, polyoxometalates (POMs) were successfully captured within downsized zeolitic imidazolate frameworks (ZIFs) using solid-state ball milling ${ }^{71}$. The ZIF-8 crystals showed porous structures and appeared as homogeneous nanoparticles with sizes $<100 \mathrm{~nm}$. In this approach, the POM cages were "pushed" inside the ZIF-8 cages through the force from mechanical grinding. The resulting downsized POMs/ZIF-8 MOFs exhibited a higher methylene blue adsorption of $126 \mathrm{mg}$ dye $\mathrm{g}^{-1}$ than the $13.3 \mathrm{mg}$ dye $\mathrm{g}^{-1}$ adsorbed by bulk POMs/ZIF-8, which shows future applicability in the uptake and delivery of bioactive molecules.

\section{Ultrasonication}

Ultrasonication is another simple and promising process to deconstruct bulk materials into small particles. This method has been widely used in the field of nanomaterials, especially in the redispersion of colloidal nanoparticles, such as the exfoliation of graphite into graphene $^{72}$ and the delamination of other 2D nanomaterials, such as MXenes ${ }^{73-75}$, and clay minerals, such as layered double hydroxides and layered transition metal oxides/hydroxides ${ }^{76}$.

Recently, ultrasonication has also been applied to produce MOF nanosheets. Neutral, crystalline MOF nanosheets that were $1.5 \mathrm{~nm}$ thick and $200-300 \mathrm{~nm}$ wide 
were delaminated from bulk MOF-2 $\quad\left[\mathrm{Zn}_{2}(\mathrm{TPA})_{4}(-\right.$ $\left.\mathrm{H} 2 \mathrm{O})_{2} \cdot 2 \mathrm{DMF}\right]$ though sonication in acetone at room temperature $^{69}$. The delamination of the layered MOF-2 crystals was possible due to the weak interlayer interaction (H-bonding) between the individual paddle-wheel $\mathrm{Zn}_{2}$ clusters and the terephthalate linker layer ${ }^{77}$. The colloidal suspension exhibited Tyndall scattering, good amine intercalation properties, and reversible benzylamine (BA) and 4-methylbenzylamine (MBA) adsorption in its $2 \mathrm{D}$ crystalline lattice. The crystallinity retention for the delaminated MOF-2 nanosheets even after subsequent amine intercalation and exchange makes them suitable candidate materials for molecular adsorption/ storage and drug delivery.

In another study, bulk POM-containing Co-1,4-bis (1,2,4-triazol-1-ylmethyl)benzene $\quad\left(\left[\mathrm{Co}_{2}\left(\mathrm{H}_{2} \mathrm{O}\right)_{4}(\mathrm{BTX})_{3}\right]\right.$ $\left[\mathrm{PMo}_{12} \mathrm{O}_{40}\right]$ ) a.k.a. POM-Co-MOF was exfoliated into atomically thick layers through a wet-ball milling approach followed by $16 \mathrm{~h}$ sonication in acetonitrile (Fig. 10a, b $)^{78}$. These particular MOF nanosheets possessed more exposed active sites than their bulk counterparts and showed potential for catalytic applications,

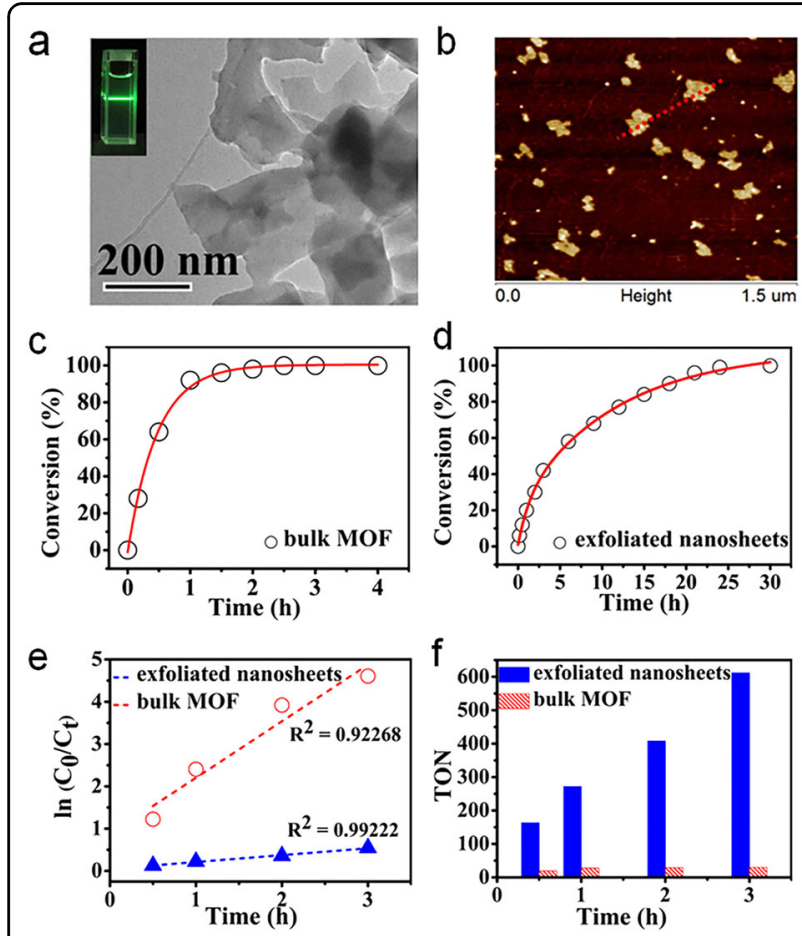

Fig. 10 Ultrasonication-assisted MOFs deconstruction into nanocrystals. a TEM and $\mathbf{b}$ AFM profiles of downsized POM-Co-MOFs. Conversion percentages of dibenzothiopehene oxidation (ODS) of c pristine and $\mathbf{d}$ delaminated POM-Co-MOF nanosheets. $\mathbf{d}$ ODS kinetics of pristine and exfoliated nanosheets. e Catalytic efficiency comparison between bulk and exfoliated catalysts. Adapted with permission from ref. ${ }^{78}$. ๑ 2018 Wiley-VCH Verlag GmbH \& Co., KGaA, Weinheim. such as oxidation and desulfurization of dibenzothiophene. When used as catalysts, the exfoliated nanosheets exhibited faster conversion of dibenzothiphene with a reaction duration of only $30 \mathrm{~h}$ than that of pristine POMCo-MOF crystals that took 4 days for the reaction to occur (Fig. 10c, d). Likewise, the turnover number (TON) of 2D-POM-Co-MOF nanosheets for the first hour of reaction is 14 times higher than that of bulk MOFs. The TON for the exfoliated MOFs increased continuously for the subsequent hours of the reaction, while it remained constant for the bulk counterpart (Fig. 10e, f). This report clearly demonstrated the improved catalytic efficiency of the exfoliated POM-Co-MOF due to its exposed active sites and improved pore accessibility ${ }^{78}$.

\section{Summary and outlook}

The variety of synthetic methodologies employed to achieve downsized MOFs is as diverse as MOF chemistry itself. Although all methods possess inherent strengths and complementarity, it is valuable to compare their impact on the resulting bulk morphology, shape, and size distribution. Insights on the scalability, yield, and efficiency are also critical. The routes presented in this review are compared against the main performance criteria summarized in Supporting Table 1.

Simplicity and scalability without the use of chemical additives are the main advantages for both kineticcontrolled approaches as well as mechanochemical techniques. In the case of kinetically controlled methodologies (shortened crystallization time and lowered temperatures), the formation of small crystals leading to low yields of crystalline products and high amounts of unreacted precursors has always been a recurring challenge due to incomplete reactions caused by an insufficient energy in the reaction system. Therefore, synthesis variables, such as the time and temperature, should be systematically adjusted and tuned to have a minimal effect on the yield and crystallinity of the products. The solvent-free nature of mechanical synthesis methods, on the other hand, offers new perspectives into convenient preparation methods and green production processes for the synthesis of nanosized crystals ${ }^{57}$. However, mechanochemical synthesis also has the same shortcomings in terms of the yield and completeness of the reaction; thus, the optimum length of the grinding time should be identified.

Other techniques, such as surfactant-assisted synthesis and the addition of coordination modulators/polymer stabilizers, provide excellent size and morphology control, as well as exceptional polydispersity and colloidal stability. These techniques have been widely used in the templatedirected synthesis of porous crystals, such as zeolites ${ }^{68}$ and mesoporous silica ${ }^{79}$. Size and morphology control was highlighted using Zn-BDC MOFs as an example ${ }^{40}$. Uniform nanorods were produced and compared to the 
irregular morphology of its bulk counterpart. Likewise, remarkable size control for HKUST-1 crystals was achieved through coordination modulation ${ }^{63}$ and ILME techniques ${ }^{44}$, where the crystals were limited from 10 to $20 \mathrm{~nm}$ and $1.6 \mathrm{~nm}$, respectively. These methods offer the potential for the tunable design of MOF materials that can be attained through the nature (e.g., shape, size, and functionality) of the micelles and/or polymer spheres during emulsification. However, the addition of surfactants also results in postsynthetic purification problems, as surfactant molecules tend to block solvent-accessible pores/channels, leading to a decreased specific surface area and loading capacity ${ }^{68}$. These techniques also require a large quantity of organic solvents that could be harmful to therapeutic cargos, thus limiting their applicability in biomedical applications.

The solvothermal method is undoubtedly the most common method employed in the synthesis of MOFs and other inorganic materials, such as zeolites. However, this approach often requires long reaction durations, which results in large crystals. In contrast, MW and US-assisted methods have shorter and more controllable reaction durations through the application of a concentrated and localized energy within the reactive mixture. An example is the shortening of HKUST- 1 synthesis from $10 \mathrm{~h}$ for the solvothermal approach down to $5 \mathrm{~min}$ for US-assisted synthesis ${ }^{6}$. Although still conducted at a laboratory scale, the combination of surfactant/modulator stabilization with MW and US-assisted methodologies clearly showed potential upscalability for industrial use due to their rapid preparation and sophisticated crystal growth control.

However, due to varying rates of formation, diverse metal-ligand/linker combinations and differences in solvation effects, additive-free kinetic-control methods and chemical-based techniques have only worked for a limited number of MOFs. This limitation for those techniques hinders their usability in large-scale synthesis reactions. Therefore, the top-down approach is attractive due to its applicability to a wide range of MOFs. Top-down strategies, such as mechanical grinding and ultrasonication, showed similarities to the mechanochemical synthesis approach in terms of the size and morphology of the final product. These methods produce nanocrystals of the desired size $(<200 \mathrm{~nm})$ that are readily usable for biomedical applications under ambient conditions in the absence of toxic solvents ${ }^{70}$. However, these techniques often result in an uncontrolled morphology due to the uneven breaking of pristine MOF crystals through physical grinding. Thus, optimization of the force used during grinding is urgently needed.

This review has critically discussed important methods that effectively led to the formation of nanoscale versions of currently existing microsized MOFs. In particular, it was highlighted that nucleation of MOF crystals may be controlled by altering the crystallization at specific stages, which produces either a reduction in the growth rate or hinders the particle growth. It is believed that this approach provides additional mechanistic insights into the scaling down of MOFs. Since not all MOFs have similar nucleation and/or growth rates or kinetics, it is unlikely that a specific downsizing method may be applicable to all types of MOFs. For example, surfactant addition or coordination modulation would only work on certain MOFs, but mechanical downsizing would be applicable to a wide range of crystal types and is less affected by the strength of the nucleation or chemistry of the ligand molecules. Therefore, summarizing different downsizing methods that influence a specific stage in MOF formation gives researchers critical options for the appropriate approach to miniaturize their MOFs (i.e., if the seeds agglomerate quickly when the solvothermal method is used, nucleation control or physical downsizing methods could be used).

\section{Acknowledgements}

The authors acknowledge financial support from the Australian Research Council (IH140100018, DP180100094, DE180100130, and FT130100380), Deakin University and the University of the Philippines (NSRI grant: CHE-16-1-07 and OPVAA/OIL-COOPERATE grant).

\section{Author details}

${ }^{1}$ Natural Sciences Research Institute, College of Science, University of the Philippines, Diliman, Quezon City 1101, Philippines. ${ }^{2}$ Deakin University, Institute for Frontier Materials, Waurn Ponds, Geelong, VIC 3216, Australia. ${ }^{3}$ Institute of Chemistry, College of Science, University of the Philippines, Diliman, Quezon City 1101, Philippines

Conflict of interest

The authors declare that they have no conflict of interest.

\section{Publisher's note}

Springer Nature remains neutral with regard to jurisdictional claims in published maps and institutional affiliations.

Supplementary information is available for this paper at https://doi.org/ 10.1038/s41427-020-00240-5.

Received: 6 January 2020 Revised: 9 June 2020 Accepted: 21 June 2020. Published online: 4 September 2020

\footnotetext{
References

1. Eddaoudi, M. et al. Modular chemistry: secondary building units as a basis for the design of highly porous and robust metal-organic carboxylate frameworks. Acc. Chem. Res. 34, 319-330 (2001).

2. Férey, G. Hybrid porous solids: past, present, future. Chem. Soc. Rev. 37, 191-214 (2008).

3. Wu, F., Qiu, L.-G., Ke, F. \& Jiang, X. Copper nanoparticles embedded in metal-organic framework MIL-101(Cr) as a high performance catalyst for reduction of aromatic nitro compounds. Inorg. Chem. Commun. 32, 5-8 (2013).

4. Wang, W. \& Yuan, D. Mesoporous carbon originated from non-permanent porous MOFs for gas storage and $\mathrm{CO} 2 / \mathrm{CH} 4$ separation. Sci. Rep. 4, 5711 (2014).

5. Lee, C. Y. et al. Light-harvesting metal-organic frameworks (MOFs): efficient strut-to-strut energy transfer in bodipy and porphyrin-based MOFs. J. Am. Chem. Soc. 133, 15858-15861 (2011).

6. Bux, H. et al. Zeolitic imidazolate framework membrane with molecular sieving properties by microwave-assisted solvothermal synthesis. J. Am. Chem. Soc. 131, 16000-16001 (2009).
} 
7. Usman, K. A. S., Buenviaje, S. C. Jr., Edañol, Y. D. G., Conato, M. T. \& Payawan, L. M. Jr. Facile fabrication of a potential slow-release fertilizer based on oxalatephosphate-amine metal-organic frameworks (OPA-MOFs). Mater. Sci. Forum 936, 14-19 (2018)

8. Anstoetz, M. et al. Novel applications for oxalate-phosphate-amine metalorganic-frameworks (OPA-MOFs): can an iron-based OPA-MOF be used as slow-release fertilizer? PLOS ONE 10, e0144169 (2015).

9. Rowsell, J. L. C. \& Yaghi, O. M. Effects of functionalization, catenation, and variation of the metal oxide and organic linking units on the low-pressure hydrogen adsorption properties of metal-organic frameworks. J. Am. Chem. Soc. 128, 1304-1315 (2006).

10. Horcajada, P. et al. Porous metal-organic-framework nanoscale carriers as a potential platform for drug delivery and imaging. Nat. Mater. 9, 172-178 (2010).

11. Usman, K. A. S., Buenviaje, S. C., Razal, J. M., Conato, M. T. \& Payawan, L. M. Synthesis and characterization of zinc adeninate metal-organic frameworks (bioMOF1) as potential anti-inflammatory drug delivery material. AIP Conf. Proc. 1958, 020018 (2018)

12. $\mathrm{Wu}, \mathrm{D}$. et al. Metal-organic frameworks as cathode materials for $\mathrm{Li}-\mathrm{O} 2$ batteries. Adv. Mater. 26, 3258-3262 (2014).

13. Flügel, E. A., Ranft, A., Haase, F. \& Lotsch, B. V. Synthetic routes toward MOF nanomorphologies. J. Mater. Chem. 22, 10119-10133 (2012).

14. Zhou, J. et al. Rational design of a metal-organic framework host for sulfur storage in fast, long-cycle Li-S batteries. Energy Environ. Sci. 7, 2715-2724 (2014).

15. Horcajada, P. et al. Flexible porous metal-organic frameworks for a controlled drug delivery. J. Am. Chem. Soc. 130, 6774-6780 (2008).

16. Furukawa, $\mathrm{H}$. et al. Ultrahigh porosity in metal-organic frameworks. Science 329, 424 (2010).

17. Xia, W. et al. Well-defined carbon polyhedrons prepared from nano metal-organic frameworks for oxygen reduction. J. Mater. Chem. A 2 11606-11613 (2014)

18. Yan, W. et al. Downsizing metal-organic frameworks with distinct morphologies as cathode materials for high-capacity Li-O2 batteries. Mater. Chem. Front. 1, 1324-1330 (2017).

19. Della Rocca, J., Liu, D. \& Lin, W. Nanoscale metal-organic frameworks for biomedical imaging and drug delivery. Acc. Chem. Res. 44, 957-968 (2011).

20. Kroll, A., Pillukat, M. H., Hahn, D. \& Schnekenburger, J. Interference of engineered nanoparticles with in vitro toxicity assays. Arch. Toxicol. 86, 1123-1136 (2012).

21. Thomas, D. G., Pappu, R. V. \& Baker, N. A. NanoParticle Ontology for cancer nanotechnology research. J. Biomed. Inform. 44, 59-74 (2011).

22. Wang, J., Byrne, J. D., Napier, M. E. \& DeSimone, J. M. More Effective Nanomedicines through Particle Design. Small 7, 1919-1931 (2011).

23. Fajula, F., Galarneau, A. \& Renzo, F. D. Advanced porous materials: new developments and emerging trends. Microporous Mesoporous Mater. 82, 227-239 (2005)

24. Cheetham, A. K., Férey, G. \& Loiseau, T. Open-framework inorganic materials. Angew. Chem. Int. Ed. 38, 3268-3292 (1999).

25. Parnham, E. R. \& Morris, R. E. lonothermal synthesis of zeolites, metal-organic frameworks, and inorganic-organic hybrids. Acc. Chem. Res. 40, 1005-1013 (2007).

26. Venna, S. R., Jasinski, J. B. \& Carreon, M. A. Structural evolution of zeolitic imidazolate framework-8. J. Am. Chem. Soc. 132, 18030-18033 (2010).

27. Jhung, S. H. et al. Microwave synthesis of chromium terephthalate MIL-101 and its benzene sorption ability. Adv. Mater. 19, 121-124 (2007).

28. Ni, Z. \& Masel, R. I. Rapid production of metal-organic frameworks via microwave-assisted solvothermal synthesis. J. Am. Chem. Soc. 128, 12394-12395 (2006)

29. Xiang, Z. et al. Facile preparation of high-capacity hydrogen storage metalorganic frameworks: A combination of microwave-assisted solvothermal synthesis and supercritical activation. Chem. Eng. Sci. 65, 3140-3146 (2010).

30. Qiu, L.-G. et al. Facile synthesis of nanocrystals of a microporous metal-organic framework by an ultrasonic method and selective sensing of organoamines. Chem. Commun. 31, 3642-3644 (2008).

31. Jung, D.-W., Yang, D.-A., Kim, J., Kim, J. \& Ahn, W.-S. Facile synthesis of MOF-177 by a sonochemical method using 1-methyl-2-pyrrolidinone as a solvent. Dalton Trans. 39, 2883-2887 (2010).

32. Zhang, B. et al. Solvent determines the formation and properties of metal-organic frameworks. RSC Adv. 5, 37691-37696 (2015)

33. Cheng, $X$. et al. Size- and morphology-controlled NH2-MIL-53(Al) prepared in DMF-water mixed solvents. Dalton Trans. 42, 13698-13705 (2013).
34. Cravillon, J. et al. Controlling zeolitic imidazolate framework nano- and microcrystal formation: insight into crystal growth by time-resolved in situ static light scattering. Chem. Mater. 23, 2130-2141 (2011)

35. Diring, S., Furukawa, S., Takashima, Y., Tsuruoka, T. \& Kitagawa, S. Controlled multiscale synthesis of porous coordination polymer in nano/micro regimes. Chem. Mater. 22, 4531-4538 (2010).

36. Schaate, A. et al. Modulated synthesis of Zr-based metal-organic frameworks: from nano to single crystals. Chemistry 17, 6643-6651 (2011).

37. Morris, W. et al. Role of modulators in controlling the colloidal stability and polydispersity of the UiO-66 metal-organic framework. ACS Appl. Mater. Interfaces 9, 33413-33418 (2017)

38. Rojas, S. et al. Nanoscaled zinc pyrazolate metal-organic frameworks as drugdelivery systems. Inorg. Chem. 55, 2650-2663 (2016).

39. Chalati, T., Horcajada, P., Gref, R., Couvreur, P. \& Serre, C. Optimisation of the synthesis of MOF nanoparticles made of flexible porous iron fumarate MIL88A. J. Mater. Chem. 21, 2220-2227 (2011).

40. Yuan, Y. et al. Surfactant-assisted facile synthesis of fluorescent zinc benzenedicarboxylate metal-organic framework nanorods with enhanced nitrobenzene explosives detection. Mater. Chem. Phys. 131, 358-361 (2011).

41. Kumar, A., Badde, S., Kamble, R. \& Pokharkar, V. B. Development and characterization of liposomal drug delivery system for nimesulide. Int J. Pharm Pharm. Sci. 2, 87-89 (2010).

42. Nune, S. K. et al. Synthesis and properties of nano zeolitic imidazolate frameworks. Chem. Commun. 46, 4878-4880 (2010).

43. Xing, T., Lou, Y., Bao, Q. \& Chen, J. Surfactant-assisted synthesis of ZIF-8 nanocrystals in aqueous solution via microwave irradiation. CrystEngComm 16, 8994-9000 (2014).

44. Zheng, W., Hao, X., Zhao, L. \& Sun, W. Controllable preparation of nanoscale metal-organic frameworks by ionic liquid microemulsions. Ind. Eng. Chem. Res. 56, 5899-5905 (2017)

45. Zhao, Y. et al. Metal-organic framework nanospheres with well-ordered mesopores synthesized in an ionic liquid/ $\mathrm{CO}_{2} /$ surfactant system. Angew. Chem. Int. Ed. 50, 636-639 (2011).

46. Bunzen, H., Grzywa, M., Hambach, M., Spirkl, S. \& Volkmer, D. From micro to nano: a toolbox for tuning crystal size and morphology of benzotriazolatebased metal-organic frameworks. Cryst. Growth Des. 16, 3190-3197 (2016).

47. Schlesinger, M., Schulze, S., Hietschold, M. \& Mehring, M. Evaluation of synthetic methods for microporous metal-organic frameworks exemplified by the competitive formation of $\left[\mathrm{Cu}_{2}(\mathrm{btc})_{3}\left(\mathrm{H}_{2} \mathrm{O}\right)_{3}\right]$ and [Cu2(btc) $\left.(\mathrm{OH})(\mathrm{H} 2 \mathrm{O})\right]$. Microporous Mesoporous Mater. 132, 121-127 (2010).

48. Klinowski, J., Almeida Paz, F. A., Silva, P. \& Rocha, J. Microwave-assisted synthesis of metal-organic frameworks. Dalton Trans. 40, 321-330 (2011).

49. Jhung, S. H., Yoon, J. W., Hwang, J.-S., Cheetham, A. K. \& Chang, J.-S. Facile synthesis of nanoporous nickel phosphates without organic templates under microwave irradiation. Chem. Mater. 17, 4455-4460 (2005).

50. Foster, B. L. \& Cournoyer, M. E. The use of microwave ovens with flammable liquids. Chem. Health Saf. 12, 27-32 (2005).

51. Son, W.-J., Choi, J.-S. \& Ahn, W.-S. Adsorptive removal of carbon dioxide using polyethyleneimine-loaded mesoporous silica materials. Microporous Mesoporous Mater. 113, 31-40 (2008)

52. Majewski, M. B., Noh, H., Islamoglu, T. \& Farha, O. K. NanoMOFs: little crystallites for substantial applications. J. Mater. Chem. A 6, 7338-7350 (2018).

53. Khan, N. A. \& Jhung, S. H. Synthesis of metal-organic frameworks (MOFs) with microwave or ultrasound: Rapid reaction, phase-selectivity, and size reduction. Coord. Chem. Rev. 285, 11-23 (2015).

54. Bigdeli, M. \& Morsali, A. Sonochemical synthesis of a nano-structured zinc(II) amidic pillar metal-organic framework. Ultrason. Sonochem. 27, 416-422 (2015).

55. Carson, C. G., Brown, A. J., Sholl, D. S. \& Nair, S. Sonochemical synthesis and characterization of submicrometer crystals of the metal-organic framework Cu[(hfipbb)(H2hfipbb)0.5]. Cryst. Growth Des. 11, 4505-4510 (2011).

56. Laybourn, A. et al. Metal-organic frameworks in seconds via selective microwave heating. J. Mater. Chem. A 5, 7333-7338 (2017).

57. Pichon, A., Lazuen-Garay, A. \& James, S. L. Solvent-free synthesis of a microporous metal-organic framework. CrystEngComm 8, 211-214 (2006).

58. Klimakow, M., Klobes, P., Thünemann, A. F., Rademann, K. \& Emmerling, F. Mechanochemical synthesis of metal-organic frameworks: a fast and facile approach toward quantitative yields and high specific surface areas. Chem. Mater. 22, 5216-5221 (2010).

59. Bian, R., Wang, T., Zhang, L., Li, L. \& Wang, C. A combination of tri-modal cancer imaging and in vivo drug delivery by metal-organic framework based composite nanoparticles. Biomater. Sci. 3, 1270-1278 (2015). 
60. Wang, F., Guo, H., Chai, Y., Li, Y. \& Liu, C. The controlled regulation of morphology and size of HKUST-1 by "coordination modulation method". Microporous Mesoporous Mater. 173, 181-188 (2013).

61. Vallet-Regí, M., Balas, F. \& Arcos, D. Mesoporous materials for drug delivery. Angew. Chem. Int. Ed. 46, 7548-7558 (2007).

62. Jin, $\mathrm{K}$ et al. [Cu(i)(bpp)]BF : the first extended coordination network prepared solvothermally in an ionic liquid solvent. Chem. Commun. 23, 2872-2873 (2002).

63. Jiang, D., Mallat, T., Krumeich, F. \& Baiker, A. Polymer-assisted synthesis of nanocrystalline copper-based metal-organic framework for amine oxidation. Catal. Commun. 12, 602-605 (2011).

64. Zhao, H.-X. et al. Theranostic metal-organic framework core-shell composites for magnetic resonance imaging and drug delivery. Chem. Sci. 7, 5294-5301 (2016).

65. Nikoobakht, B. \& El-Sayed, M. A. Evidence for bilayer assembly of cationic surfactants on the surface of gold nanorods. Langmuir 17, 6368-6374 (2001)

66. Sindoro, M., Yanai, N., Jee, A.-Y. \& Granick, S. Colloidal-sized metal-organic frameworks: synthesis and applications. Acc. Chem. Res. 47, 459-469 (2014).

67. Liao, J.-H., Wu, P.-C. \& Huang, W.-C. lonic liquid as solvent for the synthesis and crystallization of a coordination polymer: (EMI)[Cd(BTC)] (EMI = 1-Ethyl-3methylimidazolium, BTC = 1,3,5-Benzenetricarboxylate). Cryst. Growth Des. $\mathbf{6}$, 1062-1063 (2006).

68. Park, W. et al. Hierarchically structure-directing effect of multi-ammonium surfactants for the generation of MFI Zeolite nanosheets. Chem. Mater. 23, 5131-5137 (2011)

69. Li, P.-Z., Maeda, Y. \& Xu, Q. Top-down fabrication of crystalline metal-organic framework nanosheets. Chem. Commun. 47, 8436-8438 (2011).
70. Kundu, T. et al. Mechanical downsizing of a gadolinium(III)-based metal-organic framework for anticancer drug delivery. Chemistry 20, 10514-10518 (2014).

71. Li, R. et al. Polyoxometallates trapped in a zeolitic imidazolate framework leading to high uptake and selectivity of bioactive molecules. J. Mater. Chem. A 2, 2168-2173 (2014).

72. Novoselov, K. S. \& Geim, A. The rise of graphene. Nat. Mater. 6, 183-191 (2007)

73. Seyedin, S. et al. Facile solution processing of stable MXene dispersions towards conductive composite fibers. Glob. Chall. 3, 1900037 (2019).

74. Mashtalir, O., Lukatskaya, M. R., Zhao, M.-Q., Barsoum, M. W. \& Gogotsi, Y. Amine-assisted delamination of Nb2C MXene for Li-lon energy storage devices. Adv. Mater. 27, 3501-3506 (2015).

75. Naguib, M., Unocic, R. R., Armstrong, B. L. \& Nanda, J. Large-scale delamination of multi-layers transition metal carbides and carbonitrides "MXenes". Dalton Trans. 44, 9353-9358 (2015)

76. Sasaki, T. \& Watanabe, M. Osmotic swelling to exfoliation. exceptionally high degrees of hydration of a layered titanate. J. Am. Chem. Soc. 120, 4682-4689 (1998).

77. Li, H., Eddaoudi, M., Groy, T. L. \& Yaghi, O. M. Establishing microporosity in open metal-organic frameworks: gas sorption isotherms for $\mathrm{Zn}(\mathrm{BDC})$ (BDC = 1,4-benzenedicarboxylate). J. Am. Chem. Soc. 120, 8571-8572 (1998).

78. $\mathrm{Xu}$, L. et al. Exfoliating polyoxometalate-encapsulating metal-organic framework into two-dimensional nanosheets for superior oxidative desulfurization. ChemCatChem 10, 5386-5390 (2018).

79. Gao, C., Sakamoto, Y., Terasaki, O., Sakamoto, K. \& Che, S. Molecular design of the surfactant and the co-structure-directing agent (CSDA) toward rational synthesis of targeted anionic surfactant templated mesoporous silica. J. Mater. Chem. 17, 3591-3602 (2007). 